\title{
Elastic Instability of a Crystal Growing on a Curved Surface
}

\section{Citation}

Meng, Guangnan, Jayson Paulose, David R. Nelson, and Vinothan N. Manoharan. 2014. Elastic Instability of a Crystal Growing on a Curved Surface. Science 343 (6171): 634-37. doi:10.1126/ science. 1244827.

\section{Published Version}

doi:10.1126/science. 1244827

\section{Permanent link}

http://nrs.harvard.edu/urn-3:HUL.InstRepos:11859333

\section{Terms of Use}

This article was downloaded from Harvard University's DASH repository, and is made available under the terms and conditions applicable to Other Posted Material, as set forth at http:// nrs.harvard.edu/urn-3:HUL.InstRepos:dash.current.terms-of-use\#LAA

\section{Share Your Story}

The Harvard community has made this article openly available.

Please share how this access benefits you. Submit a story.

\section{Accessibility}




\title{
Elastic instability of a crystal growing on a curved surface*
}

\author{
Guangnan Meng ${ }^{1}$, Jayson Paulose ${ }^{2}$, David R. Nelson ${ }^{1,2}$ and \\ Vinothan N. Manoharan ${ }^{\dagger 2,1}$ \\ ${ }^{1}$ Department of Physics, Harvard University, Cambridge MA \\ ${ }^{2}$ Harvard School of Engineering and Applied Sciences, Harvard University, \\ Cambridge MA 02138 USA
}

One sentence summary: A constant background Gaussian curvature alters crystal growth and favors the formation of anisotropic, ribbon-like domains.

\begin{abstract}
Although the effects of kinetics on crystal growth are well-understood, the role of substrate curvature is not yet established. We study rigid, two-dimensional colloidal crystals growing on spherical droplets to understand how the elastic stress induced by Gaussian curvature affects the growth pathway. In contrast to crystals grown on flat surfaces or compliant crystals on droplets, these crystals form branched, ribbon-like domains with large voids and no topological defects. We show that this morphology minimizes the curvature-induced elastic energy. Our results illustrate the dramatic effects of curvature on the ubiquitous process of crystallization, with practical implications for nanoscale disorder-order transitions on curved manifolds, including the assembly of viral capsids, phase separation on vesicles, and crystallization of tetrahedra in three dimensions.
\end{abstract}

Since Nicolaus Steno's pioneering work on crystal growth in the 17th century (1), it has been established that the shape of a crystal is a vestige of its growth pathway. Near equilibrium, crystals grown from the melt form compact, faceted

*This is the authors' version of the work. It is posted here by permission of the AAAS for personal use, not for redistribution. The definitive version was published in Science volume 343 on February 7, 2014, DOI: 10.1126/science.1244827

†To whom correspondence should be addressed. E-mail: vnm@ seas.harvard.edu 
structures that minimize interfacial area and energy $(2,3)$, while further from equilibrium, kinetic instabilities (4) permit the formation of crystals with much larger interfacial areas, such as dendrites and snowflakes (5).

Less well-understood is the role of elastic stress, which can arise from the curvature - or lack thereof-of the space in which the crystal grows. For example, two-dimensional (2D) crystals of spheres on a spherical substrate are strained due to the incompatibility of the preferred triangular lattice packing with the Gaussian curvature of the sphere, which bends the lattice lines. Similarly, in Euclidean three-dimensional (3D) space, the absence of curvature frustrates the crystallization of tetrahedra $(9,10)$. Large, compliant crystals can alleviate this curvatureinduced elastic stress by incorporating topological defects such as grain boundary scars $(11,12)$ or pleats $(13)$ in the ground state. But for rigid crystals on curved manifolds, for which topological defects such as dislocations have large core energies, the increase of elastic stress with crystal size can also affect the growth process, such that the ground states may be inaccessible. The effects of curvature on the growth pathways are potentially important for analogous processes involving the ordering of identical subunits in curved spaces, such as the assembly of viral capsids (14), filament bundle packing (15), self-assembly of molecular monolayers (16), functionalization of nanoparticles (17), and the growth of solid domains on vesicles $(18,19,20)$.

We use confocal microscopy to examine the structures of rigid, 2D colloidal crystals growing on the inside walls of highly curved spherical water droplets (Fig. 1A). The particles start in the interior of the droplet, but over a short time nearly all of them attach to the droplet surface through a depletion attraction (6). Once at the interface, the particles attract one another through the same interaction. The short range of the attraction creates a wide coexistence region (Fig. S1) between a low-density 2D fluid and a rigid, brittle crystal phase that, unlike the 2D crystals made from repulsive particles $(11,12,13)$, cannot easily deform to cover the entire droplet. Because the high interfacial tension of the oil-water interface precludes distortion of the enclosing spherical droplets, and the depletion attraction confines particles to the droplet interface, the crystals are forced to adopt the curvature of the droplet as they grow.

This constraint has a dramatic effect on the crystal structures observed at long times: whereas 2D crystals grown on flat surfaces are compact, crystals grown on spherical surfaces are composed of slender, single-crystal segments that wrap around the droplets, as seen in Fig. 1B. In droplets with higher surface coverage, the thin segments, which we call "ribbons," join together to form branched patterns with voids and gaps between them.

If these crystals were on flat surfaces, the voids could be filled by additional particles. But the parallel transport caused by the Gaussian curvature forces the crystalline directions to be mismatched at the void borders (Fig. 2A), making it 

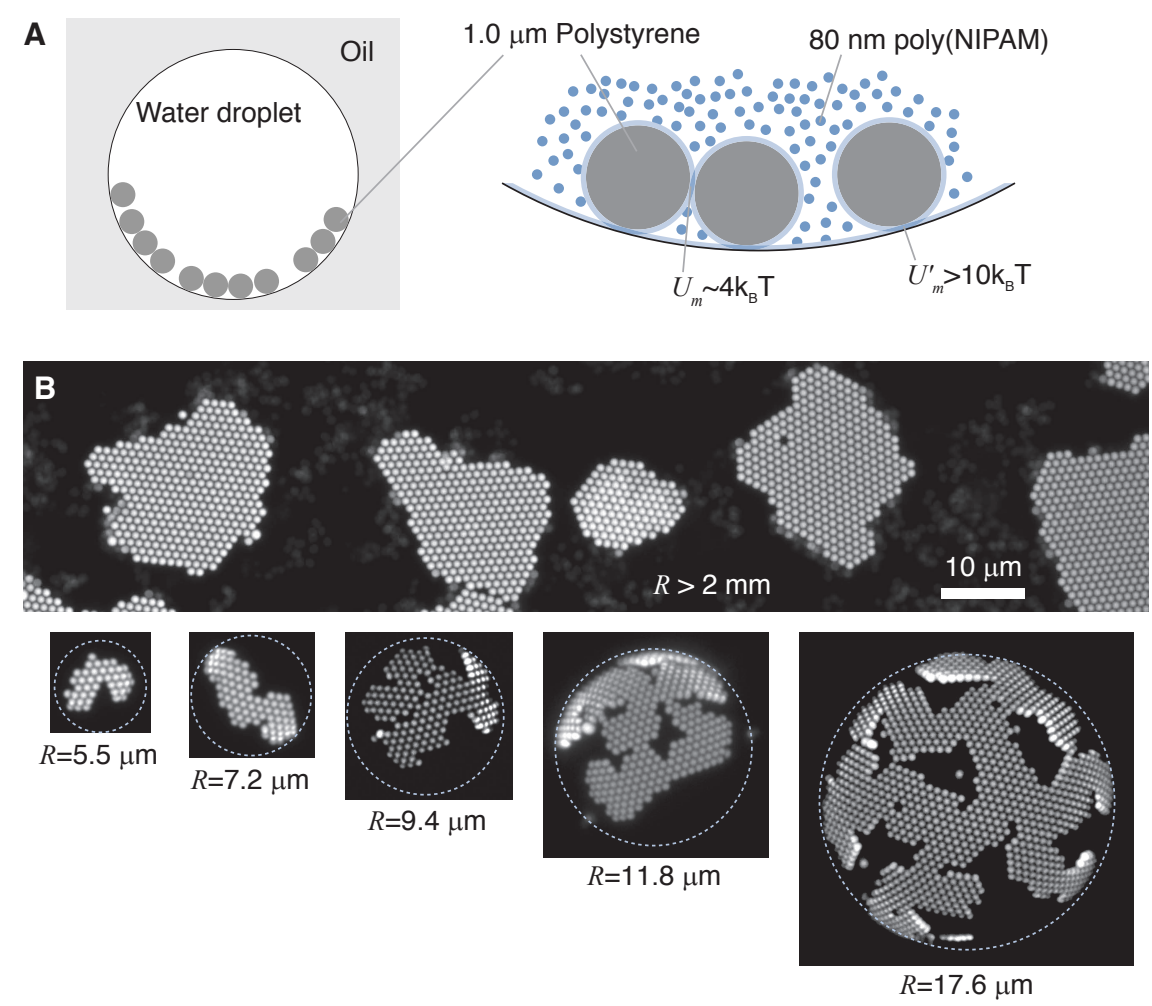

Figure 1: (A) Rigid and brittle colloidal crystals form on the interior surfaces of spherical water-in-oil droplets encapsulating an aqueous mixture of $1.0 \mu \mathrm{m}$ polystyrene (PS) spheres and $80 \mathrm{~nm}$ poly(NIPAM) particles (6). The small particles induce depletion attractions $(7,8)$ between the PS spheres with a strength of about 4 $k_{B} T$, where $k_{B} T$ is the thermal energy. The depletion force also binds the particles to the interface with a strength of about $10 k_{B} T(6)$. The range of attraction in both cases is about $80 \mathrm{~nm}$, or about $8 \%$ of the PS sphere diameter. A surfactant barrier prevents the large spheres from breaching the interface (6). (B) Representative confocal fluorescence micrographs of crystals grown for a few hours on droplets of various curvatures. Radii of curvature $R$ are noted below micrographs; dotted circles show the droplet surfaces, determined by fitting a spherical model to the particle positions. We use a very large droplet $(R>2 \mathrm{~mm})$ to approximate a flat surface. 

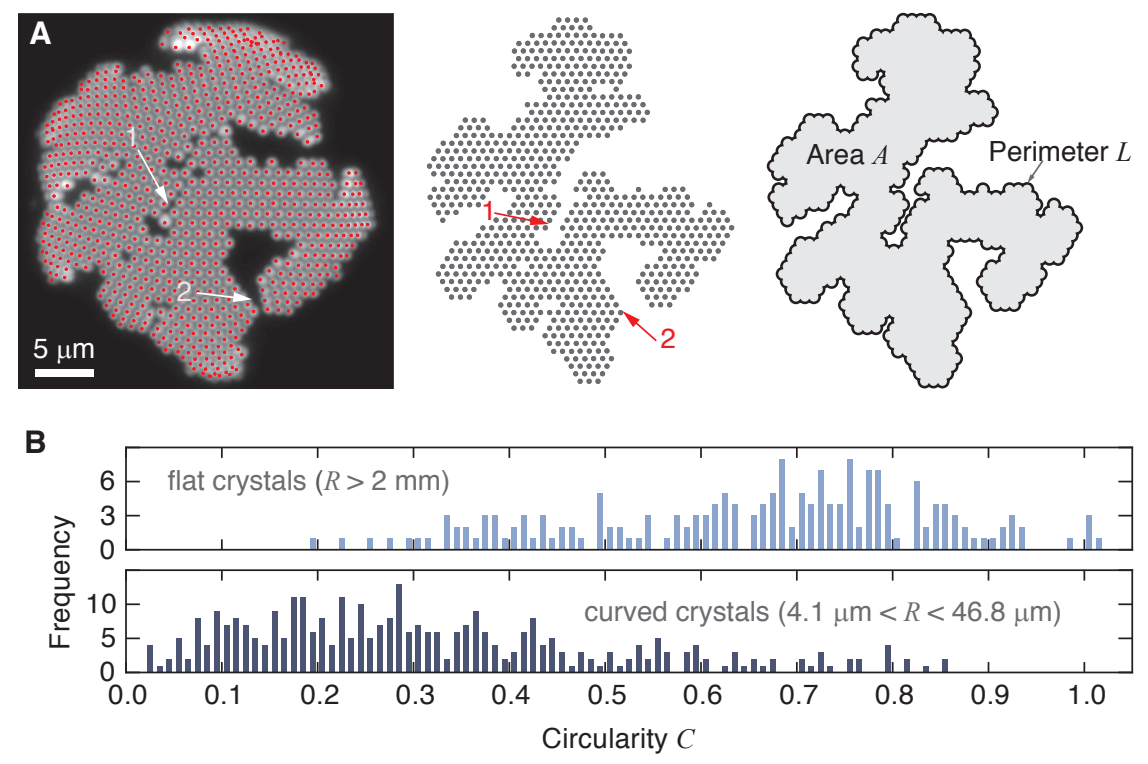

Figure 2: (A) From the 3D positions of the particles (red dots), we digitally flatten the curved crystals (6), showing that they are single grains with no topological defects. Arrow (1) shows an apparent grain boundary that is actually a row of vacancies, while arrow (2) shows a void that arises because the curvature bends the lattice vectors. (B) Histograms show that the values of the dimensionless circularity $C=4 \pi A / L^{2}$, measured using morphological image operations (6), are in general much lower for crystals grown on curved surfaces (dark blue, 335 domains) than for crystals grown on nearly flat surfaces (light blue, 187 domains).

impossible to continue the crystal without introducing topological defects. Such defects are, however, absent in our system, in contrast to curved crystals made from repulsive particles $(11,12,13)$. As we show by digitally unwrapping the crystal structures (Fig. 2A), each crystal is a single grain, and the only defects are vacancies.

Morphologically, these structures resemble dendritic crystals. We quantify the morphology using two metrics: the circularity, a measure of the perimeter-to-area ratio, and the fractal dimension, a measure of anisotropy. Crystals grown on curved surfaces have much lower circularity (Figs. 2B and S2) and fractal dimension (Fig. S3) than the compact crystals formed on flat surfaces.

In flat space, crystals with such large interfacial energies can only result from kinetic instabilities. Here, however, such instabilities are unlikely to be the cause of the anisotropy, since we do not observe dendritic crystals forming on flat surfaces under growth conditions that are otherwise identical to the curved ones (Figs. 1B and 2B). Kinetic instabilities occur when the diffusion of particles along the crystalfluid interface is slow compared to the growth rate. This happens when the domain size is comparable to the Mullins-Sekerka wavelength $\lambda_{s}(21,22)$, which is only 
weakly affected by curvature (6). We estimate $\lambda_{s}$ to be at least $100 \mu \mathrm{m}(6)$, much larger than the typical width ( $25 \mu \mathrm{m}$ or less) of our curved crystals. We therefore exclude kinetic instabilities as the cause of the morphology. We can also exclude kinetic effects arising from fluid-fluid coexistence, since our system is far from the metastable fluid-fluid critical point (6).

The remaining possibility is an elastic instability. A continuum model shows that such an instability occurs because the crystal must compress as it grows larger, owing to the Gaussian curvature of the growth surface. Consider forcing a flat, discshaped crystal of diameter $a$ onto a sphere with radius $R$. For domain sizes comparable to the sphere radius or smaller, the change in circumference-or, equivalently, the elastic strain—scales as $(a / R)^{2}$, so that the net free energy change, including the elastic energy cost, to form a circular solid domain on a curved surface is $(6,23)$

$$
\Delta G(a)=\gamma \cdot(\pi a)-\Delta f \cdot \frac{\pi a^{2}}{4}+\frac{\pi}{24576} Y \cdot \frac{a^{6}}{R^{4}},
$$

where $Y$ is the 2D Young's modulus, $\gamma$ the line tension and $\Delta f$ the chemical potential difference between the coexisting solid and fluid phases. On flat substrates $(R \rightarrow \infty)$, the elastic energy vanishes, and crystallites larger than a critical nucleus size $a_{c}=2 \gamma / \Delta f$ can grow isotropically without limit (dashed line in Fig. 3A). But on curved substrates, the elastic energy increases with domain size $a$, as measured along a geodesic. Isotropic growth becomes unfavorable beyond a critical size $a^{*} \sim(\Delta f / Y)^{1 / 4} R$ (solid line in Fig. 3A). This restriction on isotropic growth is the origin of the elastic instability (24). The predictions of this simple model are consistent with our measurements of the maximum isotropic domain size, which scales linearly with $R$ (Fig. S4A).

Although the crystal could grow larger by incorporating topological defects (25, 26), the short range of the attraction in our system makes such defects energetically costly. Geometry dictates that some interparticle distances near a five-fold or seven-fold defect are larger than the interaction range, effectively breaking the corresponding bonds. Incorporating a five-fold defect, for example, breaks five bonds. This explains the observations in Fig. 2: the stiffness of the potential favors tearing rather than stretching to accommodate stress.

Thus if the crystal is to continue to grow, it must do so by increasing its perimeter-to-area ratio. It must grow anisotropically. More specifically, the crystal should transition from a disk to a ribbon, or multiple ribbons, when its size exceeds $a^{*}$. This behavior arises because the elastic energy of a ribbon scales with the fifth power of the width $w$ but only linearly in the length $l(23,27)$. The net free energy change of forming a ribbon-like crystalline domain is:

$$
\Delta G(w, l) \approx \gamma \cdot 2(l+w)-\Delta f \cdot l w+\frac{9}{5120} Y \frac{w^{5} l}{R^{4}} .
$$



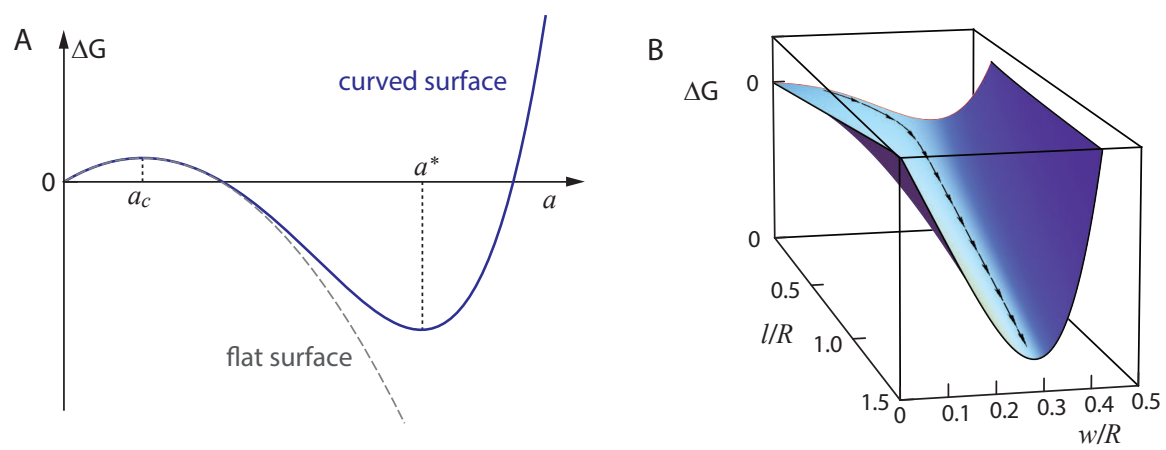

Figure 3: A continuum model shows that the elastic stress restricts isotropic domain growth to a maximum size $a^{*}$. The crystal can escape this restriction by growing anisotropically. (A) Free energy $\Delta G(a)$ for isotropic growth of a circular crystal of size $a$ on a flat surface (gray dashed line) and a surface with Gaussian curvature (blue solid line). (B) Free energy $\Delta G(w, l)$ of formation of a ribbon-shaped domain with width $w$ and length $l$. The (overdamped) crystal growth follows the gradient flow (arrows) on the energy landscape.

This energy function is shown as a landscape in Fig. 3B. By maintaining a constant width set by the curvature in the later stages of growth, the crystallite can grow to arbitrarily large lengths, limited only by the number of particles and the area available for growth. The anisotropic growth allows the crystal to avoid the size restriction imposed by elastic energy at the modest cost of a larger interfacial energy.

To test this model, we measured the dynamics of growth in single domains, as shown in Fig. 4A and Movie S1. We find that a crystal first grows isotropically until it reaches a critical size, then grows anisotropically, increasing its length while maintaining a much smaller but constant width. Examination of the final crystal shapes for several hundred droplets (Fig. 4B and S4B) shows that the length of the domains can grow to several times the droplet radius, while the width is restricted to a fraction of the radius of curvature. The near-constant value of $w / R$ seen in Fig. 4 qualitatively agrees with our model, which predicts that the elastic stress limits the width to $w \propto R$, whereas $l$ can increase without penalty. By fitting the model to the observed domain widths, we extract an effective spring constant for the interparticle interaction that is consistent with theoretical and independent experimental estimates (6), again lending support to the model.

The observation of the final, branched crystal shapes is also consistent with our physical picture. A domain can extend its length along any of the three crystallographic axes. If it changes its growth axis, it will bend by $\pm 60^{\circ}$, and if it grows in two directions at once, a branch forms. All of these growth patterns are roughly equivalent energetically, as long as the width of the growing section remains less than the critical width dictated by elasticity and curvature. Hence we expect to 
G. Meng, J. Paulose, D. R. Nelson, V. N. Manoharan, Science 343: 634 (2014)
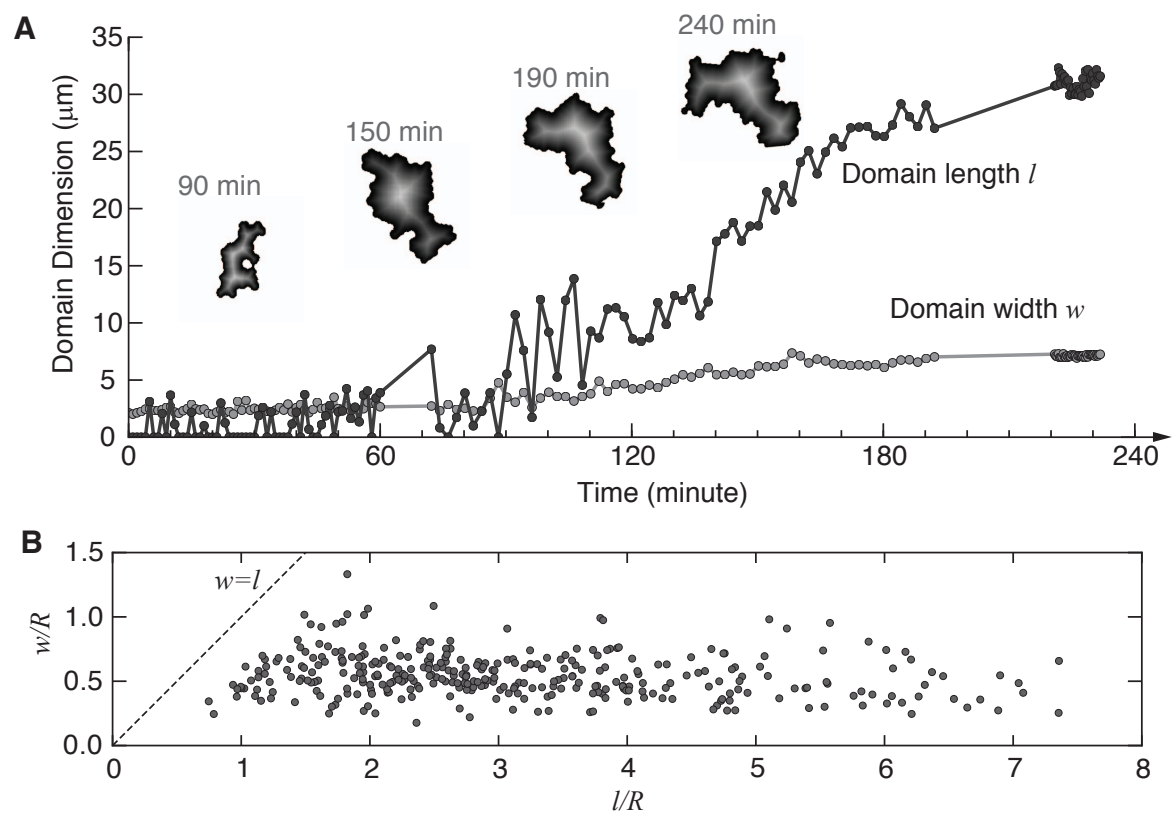

Figure 4: (A) Measurements of domain length $l$ (black) and width $w$ (gray) as a function of time for a droplet with $R=18 \mu \mathrm{m}$ (6). Images of domain morphologies at various times are shown at top (see also Movie S1). The transition from isotropic to anisotropic growth occurs at about 100 minutes. (B) Distribution of $l$ and $w$, both normalized to the radius of curvature $R$, for curved crystals. The dashed line marks the isotropic regime where $w=l . w / R$ is roughly constant, in agreement with the model. 
see, and do see, changes in direction and branches in the domains. The branches can also arise from the merger of crystallites that have nucleated independently. The branched structures resulting from such mergers maintain their curvature-dependent width $w$ in each section (see Movies S2, S3, and S4). The voids in these structures persist because the crystal directions are mismatched in adjacent sections of the crystal. In contrast, crystallites on flat substrates have no width restriction or curvature-induced mismatch and can therefore easily merge into isotropic shapes.

We conclude that anisotropic crystal growth, usually a result of a kinetic instability, can also occur by slow growth under the geometric constraint of Gaussian curvature. Our results illustrate a generic route for the growth of rigid, defect-free structures in curved spaces. This route may be particularly relevant to nanoscale substrates, where the curvature is appreciable on molecular length scales. For example, solid domains consisting of narrow stripes radiating outward from a circular core have been observed in diverse systems, from phases on lipid vesicles (20) to metal coatings on nanoparticles (17). Our analysis suggests that the width of the stripes and the core size should be determined by the interplay between curvature, elasticity and bulk energy. A similar interplay may affect the assembly pathways of viral capsids. Recent in vitro experiments (28) show that capsids can assemble following a two-step mechanism analogous to our crystallization process: the capsid proteins first attach to a substrate (an RNA molecule) and then bind together into an ordered shell. The intermediate states of this process, long a subject of speculation (14), might contain voids that, like those in our curved crystals, help the capsids avoid excess elastic stress $(29,30)$.

Similar rules may govern other crystallization and packing problems where global geometry is incompatible with local lattice packing. For example, crystallization of tetrahedra is frustrated in flat (Euclidean) 3D space (9). However, logs and helices of tetrahedra are prevalent in first-order phase transitions of tetrahedra from disordered to dense quasicrystalline phases (10). The existence of these structures, which were first described by Bernal (31), may reflect a similar physical principle, in which growth along one dimension allows a crystal to escape the restrictions of geometrical frustration. 
G. Meng, J. Paulose, D. R. Nelson, V. N. Manoharan, Science 343: 634 (2014)

\section{References and Notes}

1. N. Steno, The prodromus of Nicolaus Steno's dissertation concerning a solid body enclosed by process of nature within a solid; an English version with an introduction and explanatory notes by John Garrett Winter (MacMillan, London, 1916).

2. I. Sunagawa, Crystals: Growth, Morphology, \& Perfection (Cambridge University Press, 2005).

3. A. T. Skjeltorp, Visualization and characterization of colloidal growth from ramified to faceted structures, Physical Review Letters 58, 1444 (1987).

4. J. S. Langer, Instabilities and pattern formation in crystal growth, Reviews of Modern Physics 52, 1 (1980).

5. K. G. Libbrecht, The physics of snow crystals, Reports on Progress in Physics 68, 855 (2005).

6. Materials and methods are available as supporting material on Science Online.

7. S. Asakura, F. Oosawa, On interaction between two bodies immersed in a solution of macromolecules, The Journal of Chemical Physics 22, 1255 (1954).

8. R. Roth, B. Götzelmann, S. Dietrich, Depletion forces near curved surfaces, Physical Review Letters 83, 448 (1999).

9. D. Nelson, F. Spaepen, Polytetrahedral order in condensed matter, Solid State Physics - Advances in Research and Applications 42, 1 (1989).

10. A. Haji-Akbari, M. Engel, A. S. Keys, X. Zheng, R. G. Petschek, P. PalffyMuhoray, S. C. Glotzer, Disordered, quasicrystalline and crystalline phases of densely packed tetrahedra, Nature 462, 773 (2009).

11. A. R. Bausch, M. J. Bowick, A. Cacciuto, A. D. Dinsmore, M. F. Hsu, D. R. Nelson, M. G. Nikolaides, A. Travesset, D. A. Weitz, Grain boundary scars and spherical crystallography, Science 299, 1716 (2003).

12. P. Lipowsky, M. J. Bowick, J. H. Meinke, D. R. Nelson, A. R. Bausch, Direct visualization of dislocation dynamics in grain-boundary scars, Nature Materials 4, 407 (2005).

13. W. T. M. Irvine, V. Vitelli, P. M. Chaikin, Pleats in crystals on curved surfaces, Nature 468, 947 (2010). 
G. Meng, J. Paulose, D. R. Nelson, V. N. Manoharan, Science 343: 634 (2014)

14. W. H. Roos, R. Bruinsma, G. J. L. Wuite, Physical virology, Nature Physics 6 , 733 (2010).

15. I. R. Bruss, G. M. Grason, Non-euclidean geometry of twisted filament bundle packing, Proceedings of the National Academy of Sciences 109, 10781 (2012).

16. G. A. DeVries, M. Brunnbauer, Y. Hu, A. M. Jackson, B. Long, B. T. Neltner, O. Uzun, B. H. Wunsch, F. Stellacci, Divalent metal nanoparticles, Science 315, 358 (2007).

17. H. Bao, W. Peukert, R. K. Taylor, One-pot colloidal synthesis of plasmonic patchy particles, Advanced Materials 23, 2644 (2011).

18. R. Lipowsky, The conformation of membranes, Nature 349, 475 (1991).

19. J. Korlach, P. Schwille, W. W. Webb, G. W. Feigenson, Characterization of lipid bilayer phases by confocal microscopy and fluorescence correlation spectroscopy, Proceedings of the National Academy of Sciences 96, 8461 (1999).

20. A. Bandekar, S. Sofou, Floret-shaped solid domains on giant fluid lipid vesicles induced by pH, Langmuir 28, 4113 (2012).

21. W. W. Mullins, R. F. Sekerka, Morphological stability of a particle growing by diffusion or heat flow, Journal of Applied Physics 34, 323 (1963).

22. W. W. Mullins, R. F. Sekerka, Stability of a planar interface during solidification of a dilute binary alloy, Journal of Applied Physics 35, 444 (1964).

23. S. Schneider, G. Gompper, Shapes of crystalline domains on spherical fluid vesicles, Europhysics Letters 70, 136 (2005).

24. A. Y. Morozov, R. F. Bruinsma, Assembly of viral capsids, buckling, and the Asaro-Grinfeld-Tiller instability, Physical Review E 81, 041925 (2010).

25. Y. Chushak, A. Travesset, Solid domains in lipid vesicles and scars, Europhysics Letters 72, 767 (2005).

26. D. R. Nelson, Defects and Geometry in Condensed Matter Physics (Cambridge University Press, 2002).

27. C. Majidi, R. S. Fearing, Adhesion of an elastic plate to a sphere, Proceedings of the Royal Society A: Mathematical, Physical and Engineering Science 464, 1309 (2008). 
G. Meng, J. Paulose, D. R. Nelson, V. N. Manoharan, Science 343: 634 (2014)

28. R. F. Garmann, M. Comas-Garcia, A. Gopal, C. M. Knobler, W. M. Gelbart, The assembly pathway of an icosahedral single-stranded RNA virus depends on the strength of inter-subunit attractions, Journal of Molecular Biology advance online publication (2013).

29. W. Klug, W. Roos, G. Wuite, Unlocking internal prestress from protein nanoshells, Physical Review Letters 109, 168104 (2012).

30. A. Luque, D. Reguera, A. Morozov, J. Rudnick, R. Bruinsma, Physics of shell assembly: Line tension, hole implosion, and closure catastrophe, The Journal of Chemical Physics 136, 184507 (2012).

31. J. D. Bernal, The Bakerian lecture, 1962. The structure of liquids, Proceedings of the Royal Society of London. Series A. Mathematical and Physical Sciences 280, 299 (1964).

32. P. Robert, Temperature-sensitive aqueous microgels, Advances in Colloid and Interface Science 85, 1 (2000).

33. J. W. Goodwin, J. Hearn, C. C. Ho, R. H. Ottewill, Studies on the preparation and characterisation of monodisperse polystyrene laticee, Colloid \& Polymer Science 252, 464 (1974).

34. J. C. Crocker, D. G. Grier, Methods of digital video microscopy for colloidal studies, Journal of Colloid and Interface Science 179, 298 (1996).

35. G. Meng, N. Arkus, M. P. Brenner, V. N. Manoharan, The Free-Energy landscape of clusters of attractive hard spheres, Science 327, 560 (2010).

36. A. D. Dinsmore, E. R. Weeks, V. Prasad, A. C. Levitt, D. A. Weitz, ThreeDimensional confocal microscopy of colloids, Applied Optics 40, 4152 (2001).

37. E. Jones, T. Oliphant, P. Peterson, et al., SciPy: Open source scientific tools for Python, http://www. scipy. org (2001-).

38. E. R. Dougherty, R. A. Lotufo, Hands-on morphological image processing (Spie Press, 2003).

39. H. Xia, P. G. Tucker, Proceedings of the 18th International Meshing Roundtable (Springer-Verlag, 2009), pp. 247-265.

40. A. Vrij, Polymers at interfaces and the interactions in colloidal dispersions, Pure and Applied Chemistry 48, 471 (1976).

41. W. B. Russel, D. A. Saville, W. R. Schowalter, Colloidal Dispersions (Cambridge University Press, 1992). 
G. Meng, J. Paulose, D. R. Nelson, V. N. Manoharan, Science 343: 634 (2014)

42. M. Dijkstra, Phase behavior of hard spheres with a short-range yukawa attraction, Physical Review E 66, 021402 (2002).

43. P. Bolhuis, M. Hagen, D. Frenkel, Isostructural solid-solid transition in crystalline systems with short-ranged interaction, Physical Review E 50, 4880 (1994).

44. J. Serrano-Illán, G. Navascués, E. Velasco, L. Mederos, Self-consistent nonperturbative theory: Treatment of colloidal-type interactions, The Journal of Chemical Physics 119, 1510 (2003).

45. T. Chou, D. R. Nelson, Dislocation-mediated melting near isostructural critical points, Physical Review E 53, 2560 (1996).

46. A. Santos, M. López de Haro, S. Bravo Yuste, An accurate and simple equation of state for hard disks, The Journal of Chemical Physics 103, 4622 (1995).

47. H. Reiss, H. L. Frisch, J. L. Lebowitz, Statistical mechanics of rigid spheres, The Journal of Chemical Physics 31, 369 (1959).

48. E. Helfand, H. L. Frisch, J. L. Lebowitz, Theory of the two- and onedimensional rigid sphere fluids, The Journal of Chemical Physics 34, 1037 (1961).

49. J. A. Zollweg, G. V. Chester, P. W. Leung, Size-dependent properties of twodimensional solids, Physical Review B 39, 9518 (1989).

50. M. Engel, J. A. Anderson, S. C. Glotzer, M. Isobe, E. P. Bernard, W. Krauth, Hard-disk equation of state: First-order liquid-hexatic transition in two dimensions with three simulation methods, Physical Review E 87, 042134 (2013).

51. H. S. Seung, D. R. Nelson, Defects in flexible membranes with crystalline order, Physical Review A 38, 1005 (1988).

52. P. R. t. Wolde, D. Frenkel, Enhancement of Protein Crystal Nucleation by Critical Density Fluctuations, Science 277, 1975 (1997).

53. J. Lutsko, G. Nicolis, Theoretical Evidence for a Dense Fluid Precursor to Crystallization, Physical Review Letters 96, 046102 (2006).

54. J.-P. Hansen, I. R. McDonald, Theory of simple liquids (Access Online via Elsevier, 1990).

55. D. G. Chae, F. H. Ree, T. Ree, Radial Distribution Functions and Equation of State of the Hard-Disk Fluid, The Journal of Chemical Physics 50, 1581 (1969). 
G. Meng, J. Paulose, D. R. Nelson, V. N. Manoharan, Science 343: 634 (2014)

56. G. Tarjus, F. Sausset, P. Viot, Statistical mechanics of liquids and fluids in curved space, arXiv preprint arXiv:1005.2684 (2010).

57. S. Lishchuk, Equation of state of the hard-disk fluid on a sphere from PercusYevick equation, Physica A: Statistical Mechanics and its Applications 369, 266 (2006).

58. L. D. Landau, L. P. Pitaevskii, E. Lifshitz, A. M. Kosevich, Theory of Elasticity, Third Edition: Volume 7 (Butterworth-Heinemann, 1986).

59. We thank Frans Spaepen for helpful discussions. We acknowledge support from the Harvard MRSEC, supported by the National Science Foundation under grant number DMR-0820484. See Supplementary Materials for additional data.

Supporting Online Material

www.sciencemag.org

Materials and Methods

Figs. S1 to S4

References (32-58)

Movies S1 to S4 


\title{
Supplementary Materials for
}

\section{Elastic instability of a crystal growing on a curved surface}

\author{
Guangnan Meng, ${ }^{1}$ Jayson Paulose ${ }^{2}$ David R. Nelson, ${ }^{1,2}$ Vinothan N. Manoharan ${ }^{1,2 *}$ \\ ${ }^{1}$ Department of Physics, Harvard University, Cambridge MA 02138 USA \\ ${ }^{2}$ Harvard School of Engineering and Applied Sciences, Harvard University, Cambridge MA 02138 USA \\ *To whom correspondence should be addressed; E-mail: vnm@seas.harvard.edu
}

\section{This PDF file includes:}

Materials and Methods

Supplementary Text

Figs. S1 to S4

Captions for Movies S1 to S4

Full reference list

\section{Other Supplementary Materials for this manuscript includes the following:}

Movies S1 to S4 


\section{Materials and Methods}

\section{Preparation of colloidal particles}

The aqueous colloidal suspension consists of two types of particles, large polystyrene (PS) spheres and small poly( $N$ '-Isopropylacrylamide) (poly(NIPAM)) spheres. The poly(NIPAM) spheres induce a depletion force between the larger spheres, causing them to attract one another and stay near the water-oil interface.

We used precipitation polymerization (32) to synthesize the poly(NIPAM) hydrogel particles. $2.0 \mathrm{~g}$ of $N^{\prime}$-Isopropylacrylamide (NIPAM, monomer, 99\%, Acros Organics), $0.1 \mathrm{~g} N, N$ '-Methylenebisacrylamide (crosslinker, 99\%, Promega), and $0.19 \mathrm{~g}(\approx 6.6 \mathrm{mM})$ sodium dodecyl sulfate (SDS, 99\%, EMD Chemicals) were dissolved in $94 \mathrm{~mL}$ deionized (DI) water (Milli-Q synthesis grade, Millipore). The solution was stirred and heated to $70^{\circ} \mathrm{C}$ under nitrogen for 30 minutes to remove extra oxygen in the solution. $60 \mathrm{mg}$ of potassium persulfate (KPS, initiator, 99\%, Acros) were dissolved in $4 \mathrm{ml}$ DI water and loaded into the reactor to start the polymerization reaction. The reaction ran for 4 hours. Afterward, the poly(NIPAM) hydrogel particles were cleaned by dialysis against DI water for seven days, with water changes every 24 hours. The hydrodynamic diameter of our particles, as measured by a Zetasizer Nano ZS (Malvern Instruments), was $80 \mathrm{~nm}$ at $20^{\circ} \mathrm{C}$ and $40 \mathrm{~nm}$ at $40^{\circ} \mathrm{C}$ in DI water.

Samples observed with confocal fluorescence microscopy were prepared using $1.0 \mu \mathrm{m}$ fluorescent sulfate latex PS particles (FluoSpheres ${ }^{\circledR}$ sulfate microspheres, $1.0 \mu \mathrm{m}$, red fluorescent (580/605), Molecular Probes, Cat. No. F8851, polydispersity (standard deviation in particle diameter $=3 \%$ ). Before using, we cleaned the particles in DI water by repeated centrifugation and redispersion.

Samples observed using confocal reflection microscopy were prepared using $0.6 \mu \mathrm{m}$ non-fluorescent sulfate PS particles, synthesized by surfactant-free emulsion polymerization (33). $15 \mathrm{~mL}$ of styrene (monomer, 99\%, Sigma-Aldrich) and $146 \mathrm{~mL}$ DI water were mixed and gently stirred by a magnetic stir bar at $200 \mathrm{rpm}$, then heated to $70^{\circ} \mathrm{C}$ under nitrogen for 30 minutes. $0.1268 \mathrm{~g}$ of KPS were dissolved in $4 \mathrm{ml}$ DI water and loaded into the reactor to start the polymerization reaction. The reaction ran for 20 hours. Afterward, the PS particles were cleaned by filtration and repeated centrifugation $(3,000 \mathrm{~g}$ for 1 hour) and redispersion in DI water.

The diameter of our particles, as measured through optical microscopy (Eclipse TE-2000, Nikon Corp.), was $0.6 \mu \mathrm{m}$. In brief, a drop (about $10 \mu \mathrm{L}$ ) of colloidal suspension was dried on a glass coverslip so that the particles formed a single-crystalline monolayer. We then used image analysis (34) to locate the centers of individual particles in a focused optical micrograph. The particle size was determined by the first peak of the pair distance distribution, calculated from a few thousand particles within the single-crystalline monolayer.

\section{Emulsion preparation}

The aqueous (internal) phase of the emulsions contained a mixture of PS particles (either $1.0 \mu \mathrm{m}$ fluorescent PS or $0.6 \mu \mathrm{m}$ plain PS) and $80 \mathrm{~nm}$ poly(NIPAM) hydrogel particles in water. The concentration of PS varied; the concentration of poly(NIPAM) was $1.0 \% \mathrm{w} / \mathrm{w}$, with an estimated volume fraction of $\phi_{s} \approx 25 \%$ at $20^{\circ} \mathrm{C}$ ). $15 \mathrm{mM} \mathrm{NaCl}$ were added to screen the electrostatic repulsion between the PS particles, and 0.1\% w/w Pluronic P123 (BASF) and 0.25\% w/w Pluronic F108 (BASF) surfactant were added to stabilize the PS particles in the solution (35). The surfactants also prevented the PS particles from penetrating the water-oil interface, thus ensuring that the interaction 
between the particles was mediated entirely by the solution conditions inside the droplet, and not by interfacial effects such as capillary forces.

To prepare the emulsion, we added $100 \mu \mathrm{L}$ of aqueous solution to $2 \mathrm{ml}$ silicone oil (Alfa Aesar, Cat. No. A12728) in a glass vial and emulsified using a vortex mixer (VWR). The average size of the emulsified droplets was roughly controlled by the vortexing speed and time, and was between 20 and $50 \mu \mathrm{m}$ after vortexing at 1,500 rpm for 30 seconds.

\section{Sample cell preparation and loading}

Sample cells were prepared from silicone-treated cover slips. The silicone prevented the droplets from wetting and spreading on the cover glass. The cover slips $(22 \times 22 \mathrm{~mm}$, No.1, VWR $)$ were spin-coated at 4,000 rpm for 30 seconds with a 10\% w/w solution of silicone (10:1 mixture of base and curing agent of SYLGARD ${ }^{\circledR} 184$, Dow Corning Corporation) in cyclohexane. The coated cover slips were left in covered petri dishes at room temperature $\left(22^{\circ} \mathrm{C}\right)$ for 24 hours, during which time the silicone coating cured to form transparent, hydrophobic films a few micrometers thick.

The emulsion was then loaded between two treated cover slips. The inner edges of the glass cell were sealed with high vacuum grease (Dow Corning) of about $300 \mu \mathrm{m}$ thickness, and the outer edges were sealed by epoxy (5 Minute ${ }^{\circledR}$ Epoxy, Devcon) to prevent solvent evaporation. We waited at least 15 minutes for the glue to be fully cured before observing the samples. For measurements of the crystal structures at long times, we equilibrated the samples at room temperature $\left(22^{\circ} \mathrm{C}\right)$ for at least 24 hours before observations.

\section{Confocal microscopy observation}

We used a laser scanning confocal microscope (Leica TCS SP5 II, Leica Microsystems) equipped with an oil immersion objective $(100 X$, N.A. $=1.4$ or $63 \mathrm{X}, \mathrm{N} . \mathrm{A} .=1.4)$ to observe the three dimensional (3D) structures of the crystals (36). We did not need to index-match the particles to the medium, since there was only a monolayer of colloidal particles at the interface.

We used different observation modes depending on which PS particles were used to prepare the sample.

a. Confocal fluorescence microscopy for $1.0 \mu \mathrm{m}$ fluorescent PS particles. Laser light with a wavelength of $543 \mathrm{~nm}$ was used to excite the fluorophores in the PS particles, and emitted light was collected at $605 \mathrm{~nm}$. The figures of the main text show confocal fluorescent microscopy images.

b. Confocal reflection microscopy for non-fluorescent $0.6 \mu \mathrm{m}$ PS particles. We illuminated the sample with a 543-nm laser and collected back-scattered light at the same wavelength. Fig. SM1 shows an example of a curved crystal structure formed by $0.6 \mu \mathrm{m}$ plain PS particles, as imaged using confocal reflection microscopy.

To verify that the crystallization of the polystyrene spheres was driven by the weak depletion interaction induced by poly(NIPAM) particles, we used a microscope objective heater (MTC-HLS025, Bioscience Tools) to heat the immersion microscope objective and control the sample temperature from ambient room temperature up to $35^{\circ} \mathrm{C}$ with $\pm 0.1^{\circ} \mathrm{C}$ resolution. Since poly(NIPAM) depletants change size with temperature, the depletion attraction between PS particles is temperature sensitive: the range and strength decreases as the temperature increases. The sublimation of 


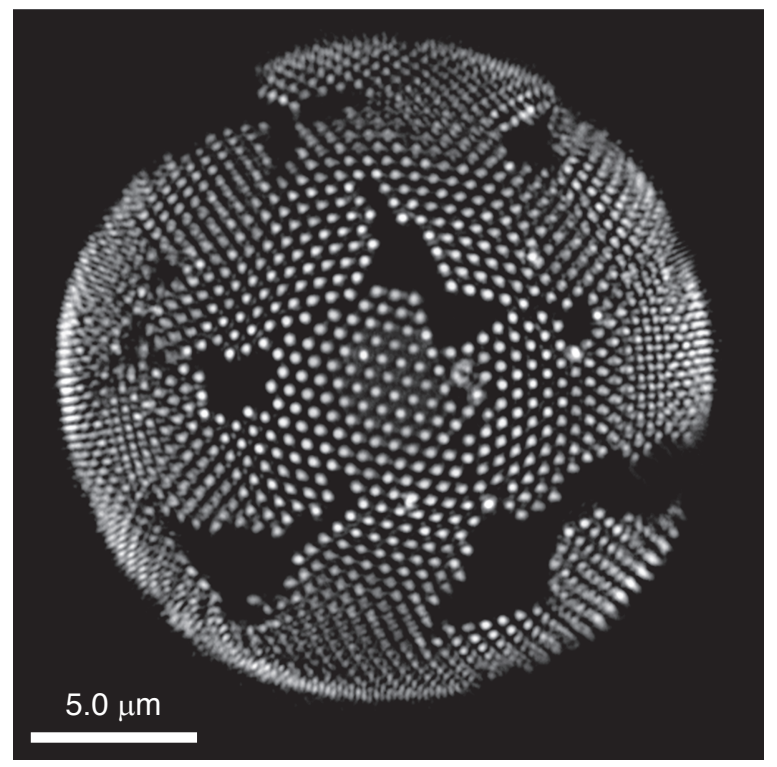

Fig. SM1. Confocal reflection microscopy of curved crystals of $0.6 \mu \mathrm{m}$ plain PS particles. The image shows the projected view of the 3D dataset along the optical axis. Scale bar, $5.0 \mu \mathrm{m}$.

the crystals at higher temperature showed that the dominant interaction between the spheres was the depletion interaction caused by the poly(NIPAM).

To show that the particles were bound to the interface by the depletion force, and not by a capillary interaction, we tracked particles in the melting experiments, and found that the PS spheres began to desorb from the interface when the sample was heated (Fig. SM2). When the sample was cooled, the particles re-adsorbed and crystallized again. Furthermore, in the absence of the poly(NIPAM) particles, we did not observe the PS particles to stick to the interface.

\section{Image processing and analysis methods}

The experimental data from the confocal microscope is structured as a 3D intensity stack, $I(x, y, z)$, stored as a 3D array of floating-point numbers. To extract information about the crystal domains, we used particle finding methods and morphology operations. This analysis was performed on Intel-based desktop computers or Linux clusters (Intel Xeon 64-bit, $2670 \mathrm{MHz}, 132 \mathrm{~GB}$ of RAM; or AMD Opteron 64-bit, $2200 \mathrm{MHz}, 198$ GB of RAM), using the Python language (http://www . python.org) with the SciPy extensions (http://www. scipy.org/) (37).

\subsection{Particle finding and domain flattening}

From the confocal stacks $I(x, y, z)$, we used a particle finding algorithm (34) to locate the centers of particles in 3D space, as shown in Fig. 2A in the main text, with a precision better than $100 \mathrm{~nm}$. Because the large majority of particles in the solid phase have six neighbors, it is possible to isolate domains of crystalline order on the sphere and flatten them onto an idealized hexagonal lattice while preserving the topology of the network of interparticle bonds. The flattened image is shown in the second panel of Fig. 2A in the main text.

To flatten the domains, we first identified nearest-neighbor pairs of particles by an adjacency criterion: two spheres were counted as neighbors if their gap distance was within the $100 \mathrm{~nm}$ depletion 


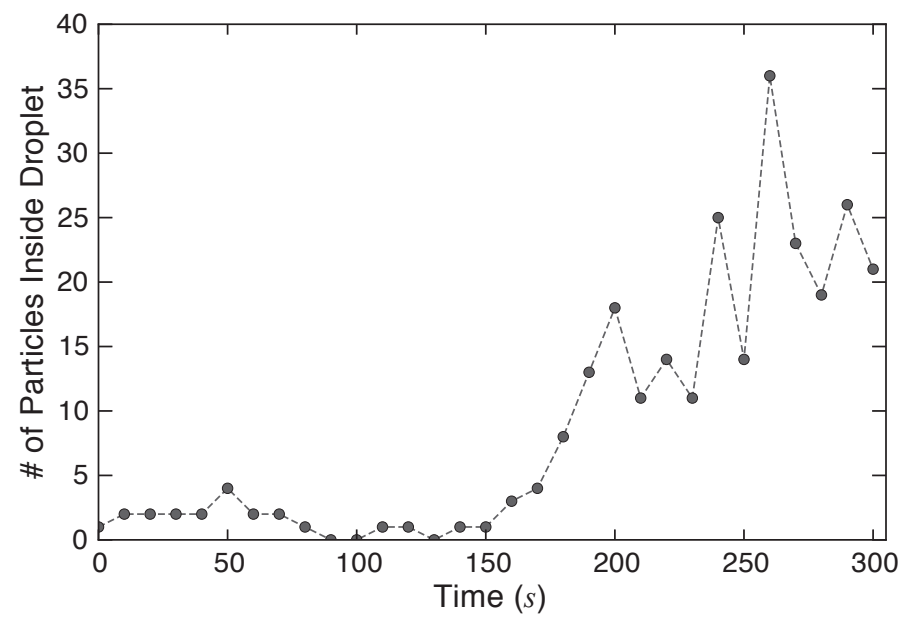

Fig. SM2. Particle dynamics during a melting experiment. From the confocal microscope stacks comprising each individual frame, we located the centers of several hundred 1.0- $\mu \mathrm{m}$ PS particles in $3 \mathrm{D}$. Then we found the center $(\mathbf{O})$ and radius of the droplet $(R)$ at each frame by least squares fitting to the distance between these particles and the droplet surface. During the melting experiment, the number of particles that were $1.0 \mu \mathrm{m}$ away or more from the droplet surface increased with time, providing evidence that the particles were bound by depletion interactions and not capillary forces.

interaction range. We then used the resulting adjacency matrix to build a graph of nearest-neighbor relationships. The graph was traversed using a depth-first search. The first pair of particles were assigned to the $(0,0)$ and $(1,0)$ lattice positions of the 2D flattened lattice. Subsequent particles were added to the lattice as follows: for every $i$ th particle visited in the traversal, the angle made by the positions of particles $i, i-1, i-2$ at $i-1$ determined the lattice position of the $i$ th point relative to the $i-1$ th particle. For example, if the link between the third and second point of the traversal made approximately a $180^{\circ}$ angle with the link between the second and first point, the third point was placed at position $(2,0)$ on the $2 \mathrm{D}$ lattice. In this manner, the flattened version of the domain was constructed particle-by-particle, preserving the local relationships between particles. The algorithm misplaces a few $(\approx 10)$ particles, especially near the outer edges and gaps in the domain. We corrected these errors by hand. The flattening process was done only to illustrate the crystalline order in Fig. 2 and was not used for any quantitative analysis.

\subsection{Morphological operations}

To extract information about the shapes of the crystalline domains we performed a series of morphological operations (38) on the confocal microscopy image data. We first interpolated the raw data to a resolution of $0.125 \mu \mathrm{m}$ per voxel along each direction using a cubic spline. Then, to find solid regions and prepare the data for further analysis, we performed the following operations (see Fig. SM3 for results of each step on the data from a representative experiment):

1. Filtering: to eliminate long-wavelength modulation of the brightness due to contrast gradients from the raw confocal stack (Fig. SM3A), we subtract a boxcar average over a volume of extent $2 w+1$ in each dimension, where $w$ is the particle radius in pixels (34). We also removed high-frequency noise from the camera and frame grabber by convolving the stack with a Gaussian kernel of width 1 voxel (34). Fig. SM3-B shows the filtered stack after these two operations. 

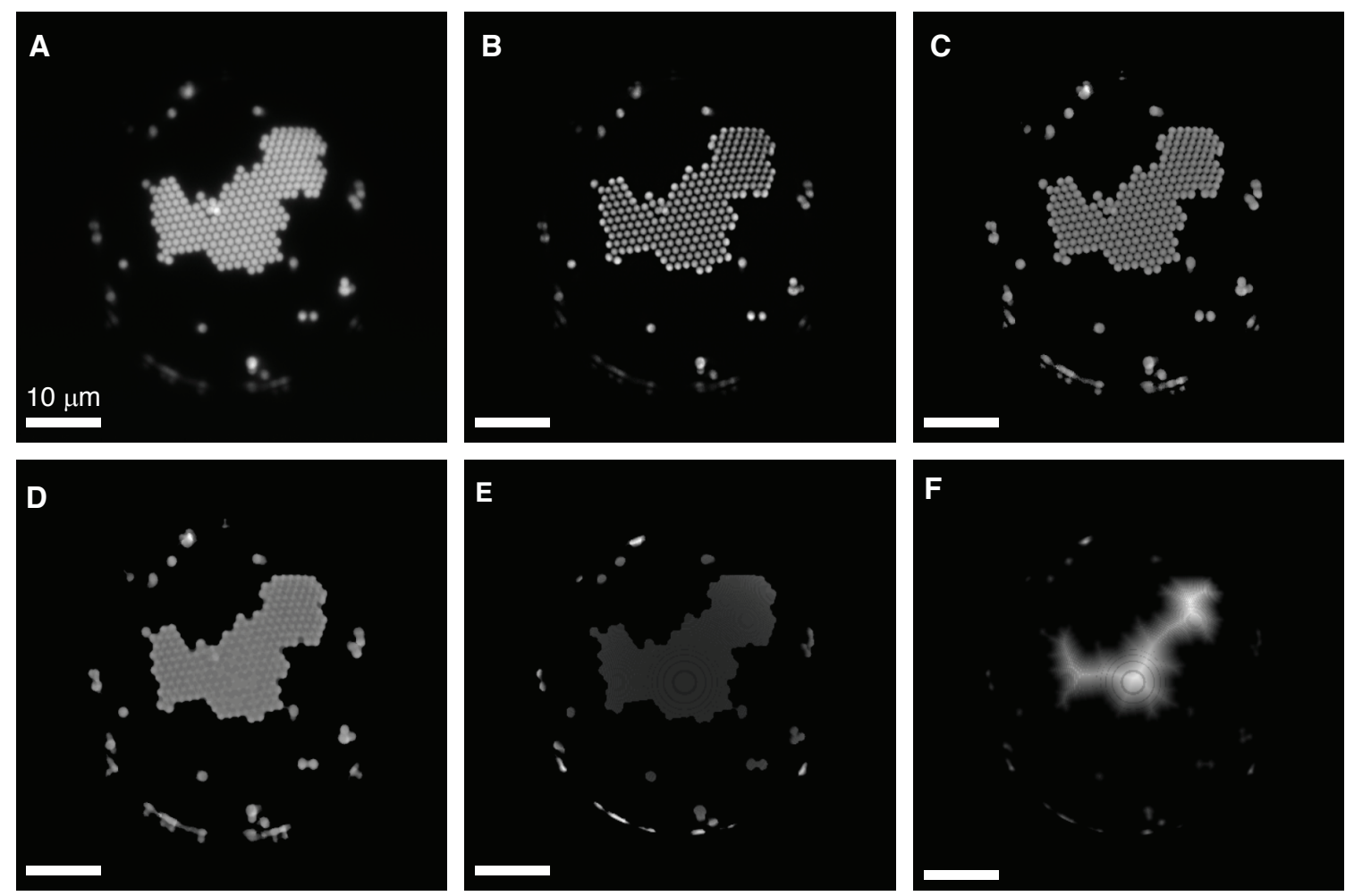

Fig. SM3. Morphological operations before further analysis (images show $z$-axis projections of 3D arrays). (A) Original confocal stack; (B) Contrast gradients and high-frequency noise removed; (C) Thresholding applied to convert stack to binary array; (D) Closing operation to fill in voids; (E) Points within $0.3 \mu \mathrm{m}$ of the droplet surface isolated; (F) Euclidean distance transform applied. Scale bar, $10 \mu \mathrm{m}$.

2. Thresholding: We then binary thresholded the filtered stack, setting all voxels above a threshold intensity to 1 and setting all other voxels to 0 . The threshold was chosen to preserve particles but eliminate low-intensity, unwanted features, such as light scattered from the droplet interface. This process results in a binary array where 1 corresponds to a region in space occupied by part of a PS particle, and 0 corresponds to empty space. Fig. SM3-C shows the binary thresholded stack.

3. Morphological closing: We performed a morphological closing operation (38) on the thresholded, binary array. This fills in all voids smaller than a single particle, and in particular fills in the regions between closely-packed particles, converting regions of crystallized particles into nearly featureless, continuous solid domains. Fig. SM3-D shows the coarse-grained stack.

4. Paring: We pared down the continuous solid domains to a uniform thickness to completely eliminate single-particle features and enable estimation of domain lengths and widths from voxel counts at later stages. First we found the center and radius of the sphere that minimizes the mean squared distance of the 1 voxels from its surface. The resulting sphere provided the best estimate of the oil-water interface, which is not directly imaged. We isolated all voxels within a finite width of $0.6 \mu \mathrm{m}$ of the droplet surface and discarded the rest. In this way, all domains had the same thickness perpendicular to the droplet surface. Fig. SM3-E shows the pared stack. 
By counting the number of voxels in each pared domain, we could estimate the size of the domain. We restricted our shape analysis to domains that covered an area corresponding to ten or more particles.

5. Euclidean distance transform (EDT): The EDT replaces each 1 voxel with the Euclidean (real space) distance from that voxel to the closest 0 voxel that lies on the droplet surface. Knowing the radius of the spherical droplet allows us to convert this Euclidean distance to a geodesic distance from each point to its closest boundary along the surface of the sphere. Fig. SM3-F shows the EDT transformed stack.

\subsection{Domain shape analysis}

We characterized the shapes of the largest crystalline domain from each droplet using several different metrics, as described below, starting from various stages of our image analysis procedure.

\subsubsection{Circularity}

The circularity $C=4 \pi A / L^{2}$ measures the perimeter-to-area ratio a solid domain, where $A$ is the domain area and $L$ is the perimeter length. $C$ measures the compactness of a solid domain relative to a disc on a planar surface (for which $C=1$ ). The smaller $C$, the more anisotropic the domain. Both area and perimeter were measured from filtered coarse-grained stacks such as the one shown in Fig. SM3-E.

The domain area $A$ was measured by counting the number of voxels within the domain:

$$
A \approx \# \text { of voxels in domain } \times \frac{\text { Volume per voxel }}{\text { Transverse thickness of domain }}
$$

where the volume per voxel is $(1.25 \mu \mathrm{m})^{3}$ (see Section 5.2).

The domain perimeter $L$ was measured by counting the number of voxels within a distance $t$ of the boundary. To smooth out features on the perimeter smaller than the scale of the particles, we chose $t$ to be the particle diameter. The number of voxels within one particle diameter of the boundary was obtained from the EDT:

$$
\begin{gathered}
L \approx \text { Number of voxels with }(\text { EDT }<\text { particle diameter }) \times \\
\frac{\text { Volume per voxel }}{\text { Transverse thickness of domain } \times \text { Particle diameter }} .
\end{gathered}
$$

These estimates of the area and perimeter for individual domains were used to calculate the circularity of crystal domains reported in Fig. 2C of the main text and Fig. S2.

\subsubsection{Mass dimension}

Another measure of the domain anisotropy is the mass dimension of the domains. The mass function $m(r)$ of a domain is the amount of material within a geodesic distance $r$ of a central point. The mass dimension $\beta$ is the exponent that describes the scaling of $m(r)$ with $r: m(r) \sim r^{\beta}$. It reflects the degree of compactness of a shape: a disc has mass dimension $\beta=2$, while an extended object such as a long, narrow rectangle, or a starfish with long arms of nearly constant width, has mass dimension $\beta=1$. On a sphere, the mass dimension is close to 2 for disc-shaped domains as long as their size is small compared to the sphere radius; it is 1 for long ribbons with width smaller than the sphere radius. 


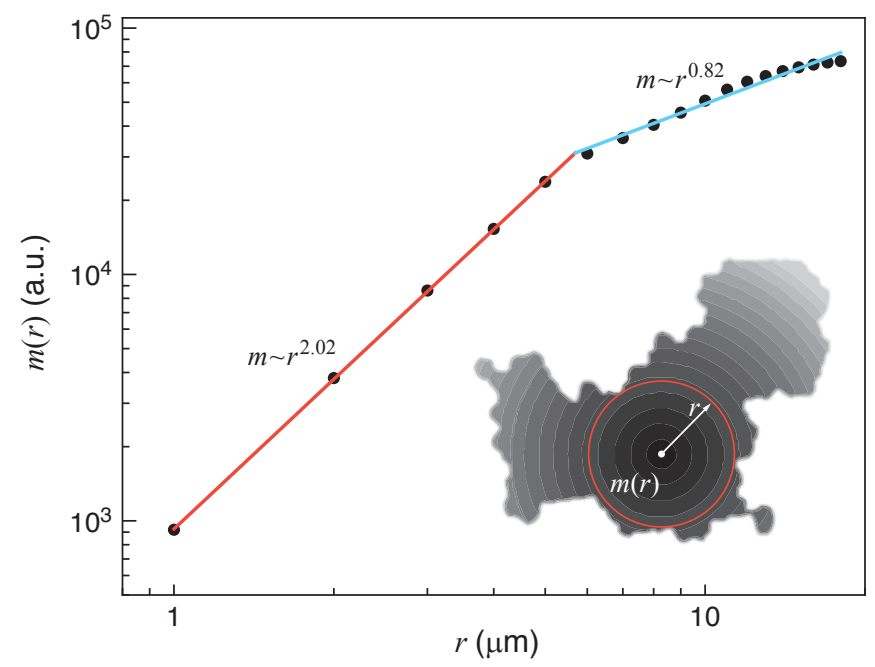

Fig. SM4. Example of a mass dimension measurement for a single domain. The mass $m(r)$ is estimated as the number of voxels within a geodesic distance $r$ of the largest inscribed disc center (white dot), with $r$ incremented by $1.0 \mu \mathrm{m}$ between data points (concentric circles in schematic). Fitting a contiguous pair of line segments to the plot gives $r_{\mathrm{I}}=5.7 \mu \mathrm{m}$ for the radius separating the inner and outer regions (see text), and the mass dimension estimates of 2.02 (red line) and 0.82 (blue line) for the inner and outer regions respectively.

We first determined $m(r)$. For each experimental domain we chose the center of the largest inscribed disc (see section 5.3.3) as the origin. The mass was estimated as the number of voxels in the solid region within a geodesic distance $r$ of the origin.

To find the mass dimension, we fit the $\log m(r)$ versus $\log r$ data to two contiguous line segments with independent slopes (Fig. SM4 solid lines). This model is based on the expected behavior of the mass dimension $\beta$ for an anisotropic or branched domain (inset of Fig. SM4): at small $r, m(r)$ should grow as $r^{2}$ until $r$ becomes comparable to the radius of the largest inscribed disc. At that point $m(r)$ must grow with a different exponent.

We used a model with four free parameters to fit the data: the intercept, the two slopes, and the distance $r_{\text {I }}$ that separates the inner and outer regions. To ensure that we identify a distinct outer region, we require that $r_{I}<r_{\max } / 2$, where $r_{\max }$ is the largest value of $r$ within the domain. Domains that fail these test are likely to be compact, with some small regions at the boundaries skewing the value of the outer mass dimension. For such domains, the outer mass dimension estimate is not reliable, and we assigned the inner mass dimension to the outer region as well.

We show $m(r)$ and a histogram of the mass dimension for several hundred domains in Fig. S3.

\subsubsection{Inscribed disc diameter, domain length, and domain width}

We measured the inscribed disc diameter $a^{*}$ of curved crystal solid domains from the EDT stack, such the one shown in Fig. SM3-F. For a given domain, $a^{*}$ is the diameter of the largest possible disc that fits completely within the solid domain. It is simply twice the largest value of the EDT. The center of the largest inscribed disc is the point in the domain with this value. Fig. SM5-A shows the center and edge of the inscribed disc for the largest domain from Fig. SM3-F.

To characterize the length and width of the non-compact and often branched or zigzag shapes of the equilibrated crystallites, we used the morphological skeleton, the set of all points in the domain 
A

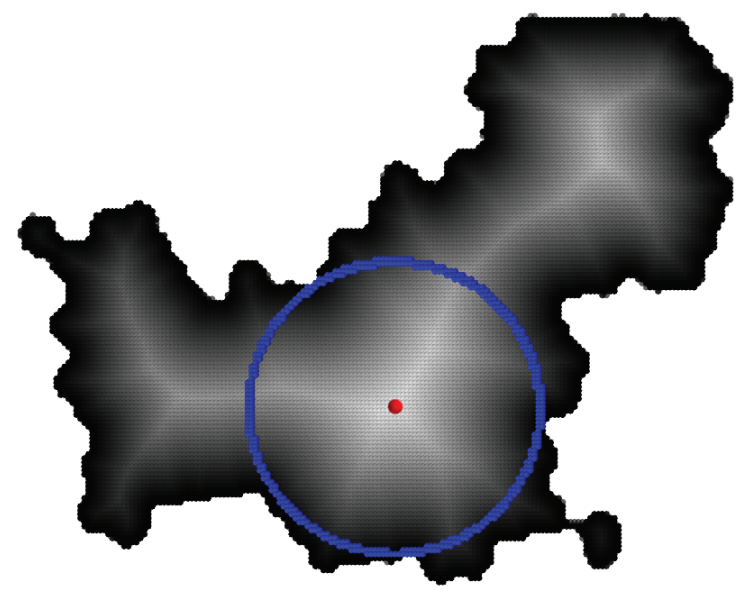

B

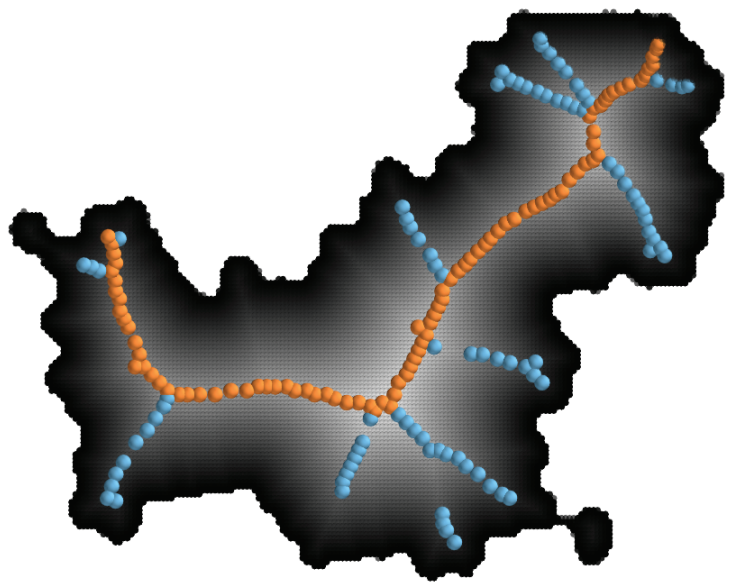

Fig. SM5. Morphological measurement of a solid domain. (A) Close-up of the largest domain from the droplet shown in Fig. SM3-F. The intensity corresponds to the Euclidean distance transform (EDT). The red dot is the point in the domain with the largest value of the EDT, which corresponds to the center of the largest inscribed disc. The blue circle shows the edge of the largest inscribed disc; the diameter of this circle (twice the largest value of the EDT) was used to estimate $a^{*}$ for the domain. (B) Morphological skeleton overlayed on the EDT. The blue and orange points follow ridges in the EDT, approximating the morphological skeleton. The orange points follow the longest contiguous branch, or backbone.

with more than one closest point on the boundary. The morphological skeleton is equivalently the set of centers of all circles that are tangent to the domain boundary at two or more points. The skeleton follows points where the Euclidean distance transform (EDT) changes sharply. It corresponds to regions where the Laplacian of the EDT takes on large negative values (39).

To estimate the morphological skeleton from the EDT of a domain, we calculated the numerical Laplacian of the EDT at each 1 voxel. We isolated voxels with Laplacian lower than a threshold of $-6.4 / \mu \mathrm{m}$, corresponding to a change in the EDT by one voxel over a distance of about 1.2 voxels. We then picked a sparse subset of these points, chosen randomly with the restriction that no two points are closer than half a particle diameter, to represent the morphological skeleton (Fig. SM5B). The value of the EDT at each skeletal point corresponds to the radius of the disc centered at that point that is tangential to two or more points on the boundary of the domain. Thus it is half the local width of the domain at that point.

A typical skeleton contains many branches, some minor branches that connect the main central branch of the skeleton to features along the boundary, and others major branches following the branched shape of the domain itself (Fig. SM5-B). To measure the length of a domain, we isolated the longest continuous branch of the skeleton by successively removing the shortest surviving branch until only two endpoints remained in the skeleton. This branch, called the backbone, is highlighted in Fig. SM5-B. For a domain that is highly convoluted but does not have any branches, the length of the backbone is a good measure of the extent of anisotropic domain growth. For a domain with multiple major branches, the length of the backbone quantifies the length of the longest section of the domain.

The length of the backbone is reported as the domain length $l$ in Fig. 4 of the main text. The average diameter of the central discs along the backbone, weighted by the area of each disc to 
minimize the effects of the tapered regions at the ends of domains, is reported as the domain width $w$ in Fig. 4 of the main text. The inscribed disc diameter $a^{*}$ and the domain width $w$ for several hundred domains are shown in Fig. S4.

\section{Theoretical methods}

\subsection{Pair potential}

Our calculations of the phase behavior and elastic energy rely on a model for the pair potential. This model has two components: a depletion attraction and an electrostatic repulsion. The driving force for the crystallization is the depletion attraction between PS particles and between the particles and the water-oil interface. We modeled the depletion interaction between two PS particles with the Asakura-Oosawa depletion theory for a binary spherical mixture $(7,40)$ :

$$
U_{A O}(r)=-k_{B} T \frac{\phi_{s}}{\left(2 a_{s}\right)^{3}}\left(2 a_{s}+2 a_{l}-r\right)^{2} \times\left(2 a_{s}+2 a_{l}+\frac{r}{2}\right)
$$

where $r$ is the center-to-center distance between two PS particles, $\phi_{s}$ the volume fraction of poly(NIPAM) depletants $(\approx 25 \%), a_{l}$ the radius of the large PS particles $\left(a_{l}=500 \mathrm{~nm}\right.$ or $\left.300 \mathrm{~nm}\right)$ and $a_{s}$ the radius of the small poly(NIPAM) $\left(a_{s}=40 \mathrm{~nm}\right)$ depletants. The range of the interaction is the diameter of the poly(NIPAM) depletant $2 a_{s}=80 \mathrm{~nm}$. The strength of the depletion attraction can be calculated when two PS particles are touching $r=2 a_{L}$ :

$$
U_{A O}\left(2 a_{L}\right)=-k_{B} T \phi_{s} \times\left(1+\frac{3 a_{l}}{2 a_{s}}\right)
$$

The surfaces of the PS particles have negatively-charged sulfate groups. When two PS particles are close to one other $\left(r \simeq 2 a_{l}\right)$, there is a screened Coulomb repulsion between them, which modifies the shape of the depletion well at small separation:

$$
U_{D L V O}(r)=k_{B} T Z^{2} \lambda_{B}\left(\frac{e^{\kappa a_{l}}}{1+\kappa a_{l}}\right)^{2} \frac{e^{-\kappa r}}{r}
$$

where $Z$ is the surface charge valence per PS particle, $\lambda_{B}$ the Bjerrum length, and $\kappa^{-1}$ the Debye screening length (41). For our experimental conditions, $\lambda_{B}=0.7 \mathrm{~nm}$, and $\kappa^{-1}=2 \mathrm{~nm}$. We do not know the surface charge for the $0.6-\mu \mathrm{m}$ plain PS particles, which were synthesized in our lab. For the fluorescent particles, we used samples from several different batches that had different surface charges as reported by the manufacturer. We therefore estimated $Z=6.4 \times 10^{4}$ for the surface charge for these beads, following our previous calculations (35, Supplementary Materials) from similarlysized particles from the same manufacturer. This surface charge is of the same order of magnitude $\left(10^{5}\right)$ as the various surface charges reported by the manufacturer for the fluorescent beads.

The pair potential between PS particles is the summation of the depletion attraction and Coulomb repulsion:

$$
\begin{aligned}
U(r) & =U_{A O}(r)+U_{D L V O}(r) \\
& =-k_{B} T \frac{\phi_{s}}{\left(2 a_{s}\right)^{3}}\left(2 a_{s}+2 a_{l}-r\right)^{2}\left(2 a_{s}+2 a_{l}+\frac{r}{2}\right)+k_{B} T Z^{2} \lambda_{B}\left(\frac{e^{\kappa a_{l}}}{1+\kappa a_{l}}\right)^{2} \frac{e^{-\kappa r}}{r} .
\end{aligned}
$$

The potential is shown in Fig. SM6. We estimate that for our experimental conditions, the strength of the depletion attraction between PS particles, taking into account the electrostatic repulsion, is $U_{m} \simeq 4 k_{B} T$. 


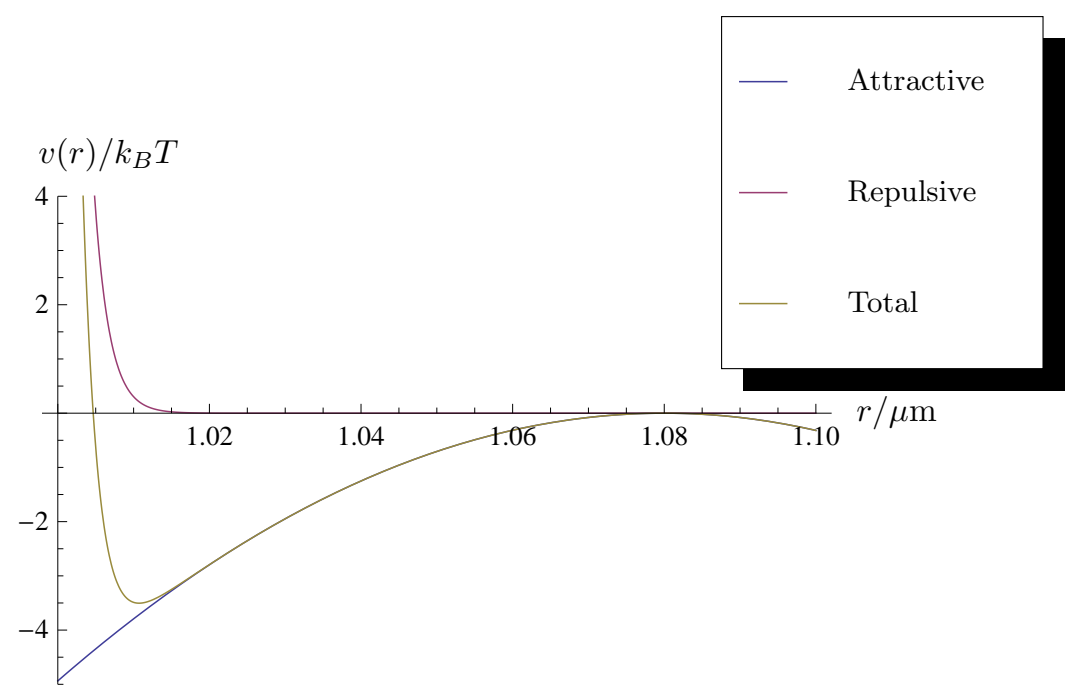

Fig. SM6. Pair potential between colloidal spheres with diameter $d=2 a_{L}=1.0 \mu \mathrm{m}$. The attractive (Asakura-Oosawa) and repulsive (screened Coulomb) components and their superposition are shown. The diameter of the poly(NIPAM) depletant particles is $80 \mathrm{~nm}$, which sets the interaction range.

The depletion attraction between the PS particles and the curved water-oil interface can be estimated in a similar manner ( 8 ). The range of the attraction is the same as between the particles, but the strength at contact is twice as large or more:

$$
U_{A O}^{\prime}\left(2 a_{L}\right)=-k_{B} T \phi_{s}\left(1+\frac{3 a_{l}}{a_{s}} \frac{R}{R-a_{l}}\right) \approx 2 U_{A O}\left(2 a_{L}\right)
$$

where $R$ is the radius of curvature, that is, the radius of the droplet that encapsulates the colloidal mixture. The depth of the attractive potential between the PS particles and droplet interface is the sum of the depletion attraction, electrostatic repulsion, and steric repulsion from surfactant molecules. Without more information on these short-range repulsive forces, we cannot estimate the well depth, but from the phase behavior and time scales for particles to dissociate from the interface, we estimate the well depth to be $U_{m}^{\prime}>10 k_{B} T$.

\subsection{Equilibrium between bulk and surface}

In our analysis, we have assumed that the crystallization is a quasi-2D process occurring from a $2 \mathrm{D}$ gas phase to a $2 \mathrm{D}$ solid phase confined to the water-oil droplet interfaces, with very little direct exchange of particles between the crystallites and the 3D interior of the droplet. This assumption is justified if practically all particles attach to the droplet interface, leaving very few particles in the interior.

Particles confined to the interface lower their energy because of the depletion interaction between each particle and the surface, but have a lower entropy because they do not access the entire $3 \mathrm{D}$ volume of the droplet. The balance between entropy and energy determines the ratio of the number $m$ of particles in the droplet interior to the number $n$ at the surface, at equilibrium. This ratio is approximately

$$
\frac{m}{n} \approx \frac{V_{\text {int }}}{V_{\text {surf }} e^{-\varepsilon / k_{B} T}} \approx \frac{1}{3} \frac{R}{\delta} e^{\varepsilon / k_{B} T},
$$


where $V_{\text {int }}$ is the volume explored by the particles in the interior and $V_{\text {surf }}$ the volume explored by particles bound to the surface. These are set by the droplet radius $R$ and the range of the interaction $\delta$ between the particles and the interface.

Our experimental results for the spring constant $k$ (see 6.6) suggest that the width of the potential well experienced by particles at the interface is $\delta \approx 10 \mathrm{~nm}$. The strength of the interaction between particles and the interface is $\varepsilon / k_{B} T \approx-10$. Hence $m / n$ varies from about 0.008 for the smallest experimental droplets with $5 \mu \mathrm{m}$ radius to about 0.08 for the largest droplets with $50 \mu \mathrm{m}$ radius. Even for the largest droplets, only a small fraction (less than 10\%) of the colloidal particles remain in the bulk, and our treatment of crystallization as a quasi-2D process is justified. Note that the attractive interactions between particles will reduce the number of particles in the bulk even more, because the particles at the interface further reduce their net free energy by forming stable crystallites. We do not observe stable crystallites in the interior volume of any of the droplets.

\subsection{D phase behavior}

To calculate the phase diagram shown in Fig. S1, we first modeled the expected phase behavior for a flat 2D system in the absence of Gaussian curvature. We consider colloidal particles interacting through the potential described in Eq. 8. Given the short range of the attraction, $\sim 0.08$ of the particle diameter, we expect any liquid-gas coexistence region to be metastable and the equilibrium phase diagram to contain a single fluid-solid coexistence region (42-44). Although hexatic phases can also appear in such systems (45), we consider only fluid and solid phases, as these are the observed phases in the experimental system. The resulting phase diagram will allow us to clarify what we mean by "rigid" versus "compliant" coexisting crystals. Although the short-range nature of the attractive part of the potential suppresses a triple point, we show that the "rigid" crystals studied here correspond to a sublimation-like region of the phase diagram.

We consider a simple, first-order model for the phase behavior. We use an equation of state for hard disks to model the fluid phase, and an Einstein solid model for the crystalline phase. These models provide analytical expressions for the free energies of both phases. To determine the phase diagram we evaluate the Helmholtz free energy per particle of both phases as a function of temperature and particle number density $\rho$. Phase coexistence is determined by equating the chemical potentials derived from these free energies.

One unusual feature of our system is the temperature-dependence of the poly(NIPAM) depletant particles, which shrink as the temperature increases, changing both $a_{s}$ as well as the depletant volume fraction $\phi_{s}$ in Eq. 8. Thus both the range and depth of the interaction potential decrease with temperature, and we expect the width of the coexistence region to decrease as well. To quantify this effect, we extract an empirical form for the depletant radius as a function of temperature $a_{s}(T)$ by fitting a polynomial to the radius measured from dynamical light scattering (Fig. SM7). Assuming that no depletant molecules enter or leave the droplet as the temperature changes, we can calculate the temperature-dependent depletant volume fraction $\phi_{s}(T)=0.25\left(a_{s}(T) / a_{s}\left(23^{\circ} \mathrm{C}\right)\right)^{3}$ from this empirical relation, using an estimated volume fraction $\phi_{s}=0.25$ at $23^{\circ} \mathrm{C}$. We use these empirical relations to calculate the temperature-dependent pair potential in our model.

\subsubsection{Fluid phase}

Because the short-ranged attraction and screened electrostatic repulsion should not significantly affect the free energy of the fluid phase at the low densities observed in the experimental system, 


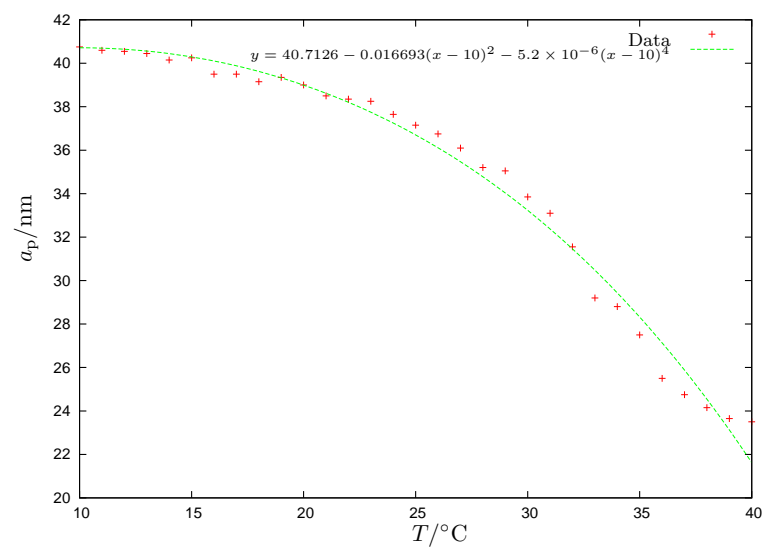

Fig. SM7. Variation of poly(NIPAM) depletant radius with temperature. The polynomial fit (green line) provides an empirical functional dependence $a(T)$ in the range $10<T /{ }^{\circ} \mathrm{C}<40$, allowing us to calculate the phase diagram as a function of temperature.

we model the fluid phase as a gas of hard disks of diameter $d$. The pair potential is

$$
v(r)= \begin{cases}\infty, & r \leq d \\ 0, & r>d\end{cases}
$$

The free energy of the fluid phase is calculated using an approximate equation of state for the hard disk fluid in two dimensions. This is expressed in terms of the compressibility factor $Z \equiv \beta p / \rho=\pi \beta p d^{2} /(4 \eta)$, with $p$ the $2 \mathrm{D}$ pressure, $\rho=N / V$ the number density corresponding to $N$ particles in a volume $V, \eta=\pi \rho d^{2} / 4$ the area fraction and $\beta=1 / k_{B} T$. For the hard-disk system, close packing corresponds to $\eta_{\mathrm{cp}}=\pi /(2 \sqrt{3}) \approx 0.907$. We use the Santos-de Haro-Yuste approximate equation of state (46),

$$
Z(\eta)=\left[1-2 \eta+\frac{2 \eta_{\mathrm{cp}}-1}{\eta_{\mathrm{cp}}^{2}} \eta^{2}\right]^{-1}
$$

which is a good approximation over a wide range of particle densities, matching the first virial coefficient exactly and also reproducing the pole in the compressibility at close packing. The Helmholtz free energy $F$ can be obtained by integrating the equation of state under constant temperature and particle number from the infinitely dilute system, which is the $2 \mathrm{D}$ ideal gas $(\rho \rightarrow 0$, $F \rightarrow F_{\text {ideal }}=N k_{B} T\left(\log \left(\rho \Lambda^{2}\right)-1\right)$ where $\Lambda=h / \sqrt{2 \pi m k_{B} T}$ is the thermal de Broglie wavelength):

$$
\begin{gathered}
F=F_{\text {ideal }}+N k_{B} T \int_{0}^{\rho} \frac{Z(\rho)-1}{\rho} d \rho \\
\beta f \equiv \frac{\beta F}{N}=\log \left(\frac{4 \eta \Lambda^{2}}{\pi d^{2}}\right)-1+\int_{0}^{\eta} \frac{Z(\eta)-1}{\eta} d \eta,
\end{gathered}
$$

where we define $f \equiv F / N$. The chemical potential, or the Gibbs free energy per particle, can be expressed in terms of the compressibility factor $Z$ :

$$
\begin{aligned}
\mu & =\frac{G}{N}=\frac{F+p V}{N} ; \\
\beta \mu & =\beta f+Z(\eta) .
\end{aligned}
$$




\subsubsection{Solid phase}

To model the chemical potential of the solid phase, we use the Einstein solid approximation, from which exact expressions of the free energy and entropy are easily obtained. We assume each colloidal particle sits in the minimum of the depletion potential well generated by six neighbors, arranged in a triangular lattice, and oscillates independently of these neighbors. Thus the $2 \mathrm{D}$ colloidal crystal can be treated as a collection of $N$ independent harmonic oscillators with frequency $\omega=\sqrt{3 k / m}$ (where $k$ is the spring constant associated with small displacements about the minimum of the pairwise interaction $U(r)$, and $m$ is the mass of the particle) and depth $\varepsilon_{0}=-3 U_{m}$. This approximation neglects the correlated fluctuations (phonons) of the solid, the fluctuations of particles normal to the interface, and the anharmonicity of the true potential. Such effects should produce only minor changes in the qualitative phase diagram calculated here.

The partition function of the crystal is then $Q_{\omega}^{N}$, where $Q_{\omega}=e^{-\beta \varepsilon_{0}} /\left(1-e^{-\beta \hbar \omega}\right)^{2}$ is the partition function of a quantum harmonic oscillator with two degrees of freedom. The harmonic oscillator decouples in the $z$ and $y$ directions, and we ignore the zero-point energy, which is minuscule compared to the well depth. Hence the Helmholtz free energy is

$$
\begin{aligned}
\beta F & =-\log \left(Q_{\omega}^{N}\right) \\
& \approx N \beta \varepsilon_{0}+2 N \log (\beta \hbar \omega), \quad \text { for } \beta \hbar \omega \ll 1 .
\end{aligned}
$$

For the Einstein solid, volume is not an independent variable, and the chemical potential can be calculated from the Helmholtz free energy:

$$
\beta \mu=\beta\left(\frac{\partial F}{\partial N}\right)_{V, T}=\beta\left(\frac{\partial F}{\partial N}\right)_{T}=\beta \varepsilon_{0}+2 \log (\beta \hbar \omega) .
$$

We rewrite this in terms of the thermal de Broglie wavelength for comparison with the fluid phase:

$$
\begin{aligned}
\beta \hbar \omega & =\frac{\Lambda}{d} \sqrt{\frac{3 k d^{2}}{2 \pi k_{B} T}} \\
\beta \mu & =\beta \varepsilon_{0}+\log \left(\frac{\Lambda^{2}}{d^{2}}\right)+\log \left(\frac{3 k d^{2}}{2 \pi k_{B} T}\right) .
\end{aligned}
$$

\subsubsection{Phase diagram}

Using approximate values for the spring constant and well depth from the theoretical pair potential, (section 6.1), we now equate the chemical potentials of the solid (Eq. 17) and fluid (Eq. 14) to determine the phase boundaries (note that the thermal de Broglie contributions cancel). Using Eq. 12 for the compressibility, we obtain $\eta_{\mathrm{f}} \approx 0.091\left(\rho_{\mathrm{f}} d^{2}=0.12\right)$ for the low-density boundary of the coexistence region at low temperature, when the depletant is at its maximum size. The density of the coexisting solid phase is very close to the close-packing density $\eta_{\mathrm{s}} \approx 0.9$, as we have assumed a hexagonal Einstein solid with the particles at the minima of the depletion interaction, which gives rise to an interparticle distance of $1.02 d$. The low density of the fluid phase is in qualitative agreement with the experiments, where nearly all particles are observed to be in the solid phase at low temperature. Using other approximations for the hard disk equation of state in place of Eq. 12, such as the approximations in refs. (47) and (48), do not significantly change the results of this approximate theory.

As the temperature increases, the coexistence region shrinks due to the change in depletant size, as shown in Fig. S1. Again, this is in qualitative agreement with experiments, which show that the system sublimates as the temperature increases. 
In this phase diagram, we have assumed that the density of the solid phase remains that of a hexagonal lattice with lattice constant equal to the position of the minimum in the depletion potential, which depends weakly on $a_{s}$. However, the stable solid phase at high temperature, when the depletion interaction is weak and even more short-ranged, may be stabilized by entropy rather than the depletion attraction. For reference, computer simulations of the melting transition (49) of the pure hard-disk system (with no attractions) show that there is coexistence between a fluid and entropic crystal between $\eta_{\mathrm{f}}=0.697\left(\rho_{\mathrm{f}}=0.887 / d^{2}\right)$ and $\eta_{\mathrm{s}}=0.710\left(\rho_{\mathrm{s}}=0.904 / d^{2}\right)$. Thus at temperatures greater than about $35^{\circ} \mathrm{C}$, there could exist a coexistence between two solid phases, a dense crystal with $\eta_{\mathrm{s}} \approx 0.9$ and an entropic crystal with $\eta_{\mathrm{s}} \approx 0.7$ (43), but we have not modeled the equilibrium between solid phases in the phase diagram shown in Fig. S1. We note in passing that three different simulation methods have recently revealed a first-order liquid-hexatic transition in the high-density regime for hard disks (50).

\subsubsection{Rigidity of the solid phase}

At temperatures below the volume phase transition of the poly(NIPAM) depletant particles - where the depletion force has its maximum depth, and the coexistence region is broad - we expect the crystals that form to be rigid due to the short range of the depletion potential. For a hexagonal network of springs of spring constant $k$, the the Lamé elastic constants are $\lambda=\mu=\sqrt{3} k / 4$ (51). At low temperature, the effective spring constant for our crystals is on the order of $10^{6}-10^{7} k_{B} T / \mu \mathrm{m}^{2}$ (section 6.6). Thus the elastic constants are large (see section 6.4.2), and the crystal is rigid. The Kosterlitz-Thouless parameter,

$$
K=\frac{4 \mu(\mu+\lambda)}{2 \mu+\lambda} \frac{b^{2}}{k_{B} T}
$$

where the Burgers vector $b \sim d$, is many orders of magnitude larger than $16 \pi$, the value at which the solid becomes stable against the formation of dislocations. The melting transition to an intermediate hexatic phase is therefore suppressed in favor of a sublimation directly into the fluid phase.

At higher temperature, the crystal should become less rigid because the spring constant decreases as the depletants shrink. A compliant solid may exist at high temperature if an entropic crystal is favored over a crystal formed by depletion bonds. See ref. (50) for a progress report on understanding the phase behavior in this hard-disk-like regime.

\subsubsection{Metastable fluid-fluid coexistence}

Finally, we calculate the fluid-fluid coexistence curve. Although the short range of attractions in the system makes any fluid-fluid coexistence metastable relative to the fluid-solid phase separation identified in Section 6.3.3, the position of the fluid-fluid coexistence curve can affect the kinetics of phase separation. Close to a fluid-fluid critical point or in a region of fluid-fluid coexistence, crystal growth may occur via a two-step nucleation mechanism $(52,53)$, where the dilute gas densifies into a dense droplet, within which the solid phase nucleates.

To estimate whether such mechanisms are relevant in the experiments, we calculate the metastable fluid-fluid coexistence curve in the 2D phase diagram, again in the absence of Gaussian curvature. To do so, we must include the effect of attraction between particles in the fluid phase, since there is no fluid-fluid phase separation in the $2 \mathrm{D}$ hard-disk fluid. To improve upon our approximate calculations in Section 6.3.1, we include the correction due to the attractive interactions to lowest order using perturbation theory (54). The reference system for our perturbation theory is the 2D hard-disk fluid, with Helmholtz free energy per particle $f_{\text {ref }}$ given by Eq. 13. The approximate free 


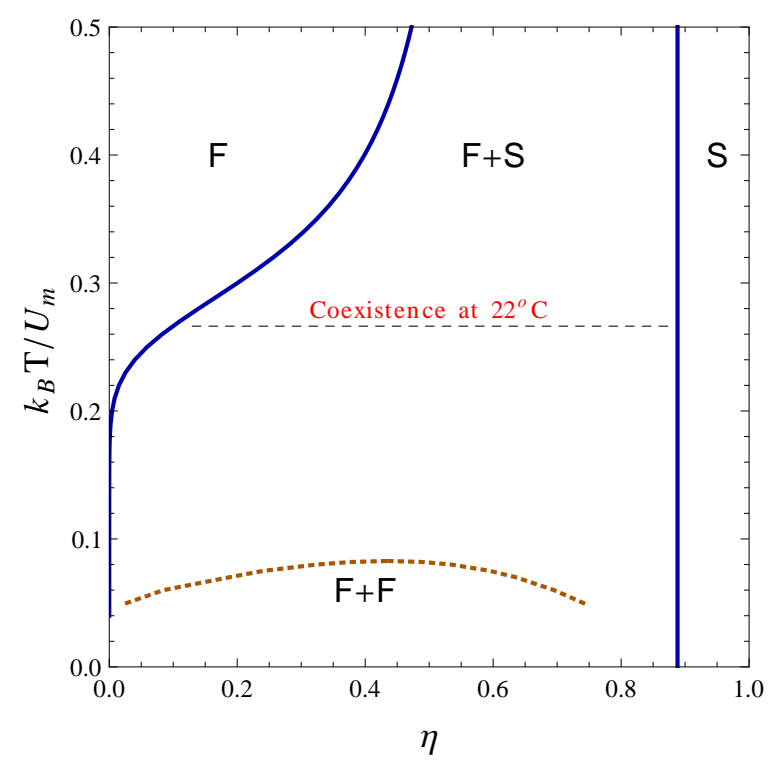

Fig. SM8. Approximate phase diagram of a 2D system of particles experiencing a fixed pair potential set by the size and volume fraction of depletant at $22^{\circ} \mathrm{C}$. In addition to the fluid-solid coexistence region, there is a metastable fluid-fluid coexistence region indicated by the dotted curve. The dashed horizontal line shows the temperature at which crystallization takes place in the experiments.

energy including the perturbative correction is (54)

$$
\beta f \approx \beta f_{\text {ref }}+\frac{\rho}{2} \int d \mathbf{r} g_{\text {ref }}(r)\left[\beta u_{\text {int }}(r)\right]
$$

where $g_{\text {ref }}$ is the radial distribution function of the hard disk reference system, and $u_{\text {int }}(r)$ is the perturbation potential which we take to be the attractive part of $U(r)$. In our calculations, we use a density expansion to order $\rho^{3}$ for $g_{\text {ref }}(r)$,

$$
g_{\text {ref }}(r) \approx \begin{cases}0, & r<d \\ 1+\rho g_{1}(r)+\rho^{2} g_{2}(r)+\rho^{3} g_{3}(r), & r \geq d\end{cases}
$$

where the $g_{i}(r)$ are numerically interpolated from the discrete values reported in Ref. (55). Using Eqs. 13 and 20 in Eq. 19, we obtain the Helmholtz free energy per particle in the fluid phase. The chemical potential, or the Gibbs free energy per particle, is:

$$
\mu(\eta)=\frac{F+p V}{N}=\frac{\partial(\eta f)}{\partial \eta} .
$$

Fluid-fluid coexistence occurs when the theoretical free energy first becomes concave. The coexistence densities are obtained by the usual Maxwell double-tangent construction.

Including the perturbative effect of interactions in the fluid free energy does not significantly change the fluid-solid coexistence curves, justifying the use of the 2D hard-disk fluid free energy to calculate Fig. S1. Significantly, our model does not show fluid-fluid coexistence for interaction potentials corresponding to the experimentally accessible poly(NIPAM) depletant radius shown in Fig. SM7. A second fluid phase (signalled by $\partial \mu / \partial \eta$ taking on negative values for some range of $\eta$ ) exists only when $a_{s}>54 \mathrm{~nm}$, larger than the maximal temperature-dependent poly(NIPAM) radius 
in the experimental system. According to our model, the interactions at the maximal poly(NIPAM) radius of $40.7 \mathrm{~nm}$ are not strong enough even for a metastable fluid-fluid coexistence.

Every temperature value in Fig. S1 is associated with a unique interaction potential. To gauge the effect of possible metastable phases on crystal nucleation, we also calculate the theoretical phase diagram for the specific interaction potential at which the experiments are conducted, which corresponds to $U(r)$ calculated using $a_{s}$ and $\phi_{s}$ at the crystallization temperature of $22^{\circ} \mathrm{C}$. As shown in Fig. SM8, crystallization in our experiments occurs far above the fluid-fluid coexistence region ( $T / T_{\mathrm{c}} \approx 3.2 \gg 1$, where $T_{\mathrm{c}}$ is the temperature of the fluid-fluid critical point). Therefore, the effect of the metastable phases on the crystallization pathway is expected to be small.

\subsubsection{Influence of curvature on the theoretical phase diagrams}

In our model, the primary effect of the curvature is to strain the crystal. We capture the effect of this strain on the free energy by adding an elastic energy penalty that depends on the shape of the solid domain, the curvature, and the effective spring constant of the pairwise interactions between particles. Here we show that we can neglect the effects of curvature on the interactions and the entropic contributions to the free energy.

Because of the small size of the depletant, there is no direct influence of the droplet interface, and therefore of its curvature, on the pairwise depletion attractions. Furthermore, there should be no significant three-body contribution to the particle-particle interactions. The Asakura-Oosawa interaction arises due to the overlap of regions from which the depletant is sterically excluded. The overlap of excluded volumes of two colloidal particles extends to at most $\sqrt{a_{s}^{2}+2 a_{l} a_{s}}=0.20 \mu \mathrm{m}$ for $2 a_{l}=1.0 \mu \mathrm{m}$ particles and $0.16 \mu \mathrm{m}$ for $2 a_{l}=0.6 \mu \mathrm{m}$ particles, and does not overlap with the excluded volume near the droplet interface in either case. Three-body interactions occur only if the excluded volumes of three particles mutually overlap; the smallest depletant radius for which this happens is $a_{l}(2 / \sqrt{3}-1)=0.077 \mu \mathrm{m}$ for $1.0 \mu \mathrm{m}$ particles and $0.046 \mu \mathrm{m}$ for $0.6 \mu \mathrm{m}$ particles, larger than the radius of the depletant in our system. Furthermore, the curvature-induced strain is not large enough to cause three-body overlaps. The maximum curvature-induced strain in the solid domains, as estimated from the domain widths, is of order $(w / R) / 8 \sim 0.002$, smaller than the ratio of the potential well width to particle size, which is of order 0.01 .

The entropic contribution to the free energy of the fluid phase, modeled using the hard-disk fluid, is also not significantly modified by confining the disks to the surface of a sphere (56) (this is in contrast to surfaces of constant negative curvature, such as the hyperbolic surface, on which the behaviour of simple fluids can differ significantly from the flat plane (56).) A scaled particle theory approximation of the compressibility factor $Z(\eta)$ for hard disks on the surface of a sphere (57) shows deviations of less than $1 \%$ for $\eta \leq 0.5$ when the sphere radius is five times the particle diameter. For spheres with radii ten times the particle diameter, the deviations are less than $0.1 \%$.

\subsection{Nucleation and growth on curved surfaces}

In this section we show how we calculate the free energy of crystal growing on the surface of the sphere, as shown in Fig. 3 of the main text.

\subsubsection{Classical theory of crystallization}

In classical nucleation theory (CNT), the free energy change of crystallization includes interfacial energy and bulk energy. The free energy change of creating a 2D crystallite of area $A$ and interfacial length $L$ is

$$
\Delta G=\gamma \cdot L-\Delta f \cdot A
$$


where $\gamma$ is the interfacial tension associated with creating a solid-gas interface of unit length, and $\Delta f>0$ is the excess chemical potential per unit area in the gas phase compared to the solid phase. For a 2D circular disk of diameter $a, L=\pi a$ and $A=\pi a^{2} / 4$. Rough estimates of $\gamma$ and $\Delta f$ can be obtained from the pair potential between individual particles, characterized by a well depth $U_{m}$ and equilibrium separation $d$. For our experimental system, $d$ can be taken to be the diameter of the PS particles, since the depletion interaction is short-ranged.

The interfacial tension is estimated by considering the energy cost for a solid domain to break enough bonds to form a free boundary per unit length along the solid-fluid interface. It corresponds to a particle breaking one bond $\left(U_{m}\right)$ over a length of one particle size $(d)$ along the interface. This gives the estimate of interfacial tension as:

$$
\gamma \approx U_{m} / d
$$

The free energy change between solid and gas phases per unit area is estimated from the chemical potential (the free energy per particle) in the solid phase. Each particle in the interior of the solid phase occupies the area of a regular hexagon with side $d / \sqrt{3}$. From the Einstein solid estimate of the chemical potential in the solid phase, Eq. 17, we estimate $\Delta f$ to be

$$
\Delta f \approx 2 \sqrt{3} \frac{U_{m}}{d^{2}}-\frac{2}{\sqrt{3}} \frac{k_{B} T}{d^{2}} \log \left(\frac{3 k d^{2}}{2 \pi k_{B} T}\right) .
$$

This estimate ignores entropic contributions from the fluid phase (aside from the trivial cancellation of the thermal de Broglie contribution), which depends on the the density of particles during crystallization. In the early stages of growth, when most particles are in the fluid phase and the fluid density is moderate, the entropic contribution is small and $\Delta f$ remains positive (i.e. solidification is favoured) due to the dominant contribution of the depletion attraction in the solid phase (Eq. 24). However, as crystallization proceeds, the number of fluid particles and hence the fluid density becomes lower, increasing the entropic contribution to the gas phase free energy and decreasing $\Delta f$. At very low fluid densities $(\eta \ll 1)$, the entropic contribution dominates the contribution from Eq. 24, which is no longer accurate; $\Delta f$ can even be zero or negative. Therefore, our rough estimate of the free energy change is valid only in the initial stages of growth. Growth stops when $\Delta f \rightarrow 0$, or when there are no particles left in the fluid phase.

\subsubsection{Elastic energy of deformation}

On a flat surface, the PS particles can assemble in a perfect hexagonal lattice, with all bonds between particles at the equilibrium length of the depletion interaction. However, on a surface of nonzero Gaussian curvature, such as a sphere, geometry dictates that the lengths of all the bonds cannot be preserved, and they must undergo distortions from their equilibrium positions. To calculate the energy cost of changing bond lengths, we approximate each bond as a harmonic potential with spring constant $k$ and with equilibrium bond length the same as that of the depletion interaction. In the continuum limit where the bond lengths are small compared to the size of the bond network, the deformation energy of the hexagonal network is equivalent to the elastic energy of deforming a two-dimensional membrane with a 2D Young's modulus $Y$ and Poisson ratio $v$ given by (51):

$$
Y=\frac{2}{\sqrt{3}} k ; \quad v=\frac{1}{3} .
$$

Within this continuum description, the elastic energy of a membrane is given in terms of the $2 \times 2$ strain tensor $u_{i j}(58)$ :

$$
E_{s}=\frac{1}{2} \int d^{2} r \frac{Y}{2\left(1-v^{2}\right)}\left[\left(u_{11}+u_{22}\right)^{2}-2(1-v)\left(u_{11} u_{22}-u_{12}^{2}\right)\right]
$$


where the integral is over the surface of the undeformed patch. To find the elastic energy of deforming a membrane patch of a particular shape on a sphere of radius $R$, we must solve the variational problem of finding the strain fields that minimize the elastic energy, subject to the boundary conditions of zero line stresses perpendicular to the boundaries, so that the displacements of the patch bring it to the required spherical shape. This is a difficult problem to solve for patches of arbitrary shape, but it has been solved for some specific shapes.

For disc-shaped elastic domains of diameter $a$, the elastic energy cost (23) is:

$$
E_{\mathrm{disc}} \approx \frac{\pi}{24576} Y R^{2}\left(\frac{a}{R}\right)^{6}
$$

For rectangular domains with length $l$ much larger than width $w$, an approximate expression for the elastic deformation energy on a sphere (27) is:

$$
E_{\text {ribbon }}=\frac{1}{640\left(1-v^{2}\right)} Y R^{2}\left(\frac{w^{5} l}{R^{6}}\right) \approx \frac{9}{5120} Y R^{2}\left(\frac{w^{5} l}{R^{6}}\right)
$$

for $v=1 / 3$. We use Eq. 27 and Eq. 28 to estimate the elastic energy cost of stretching a solid domain on the curved surface in the main text.

\subsection{Kinetic growth instability calculations}

In the main text we rule out kinetic instabilities as a cause of the observed anisotropic crystal shapes by comparing growth on curved and flat substrates and estimating the relevant physical parameters. Here we describe the details of this calculation.

Growth instabilities arise when diffusion of the fluid particles to the crystal-fluid interface is the rate-limiting step in crystal growth, which is true for large crystals. Since it takes fluid particles longer to diffuse around the interface for larger crystals, smoothing out protuberances in the growing interface may be kinetically prohibited for large crystals even if energetically favored. This leads to a dendritic growth instability for crystallites larger than a certain size. Our flat crystals, which are up to $40 \mu \mathrm{m}$ in diameter, do not exhibit dendritic growth. Since the growth conditions and particle concentrations are similar in our experiments on flat and curved substrates, we do not expect dendritic growth to be observed up to these sizes on curved substrates either.

This argument can be made quantitative by estimating the characteristic crystal size at which growth instabilities become important. A simple physical model for growth instabilities leading to dendrites is the linear stability analysis of Mullins and Sekerka $(4,21,22)$. A flat crystal-fluid interface growing into the fluid phase is expected to be unstable to perturbations of wavelength longer than the Mullins-Sekerka wavelength

$$
\lambda_{s}=2 \pi \sqrt{l_{d} l_{c} / 2}
$$

where $l_{d}$ is the macroscopic diffusion length and $l_{c}$ is the microscopic capillary length. $l_{d}=2 D / v$ for fluid particles with diffusion constant $D$ diffusing to an interface growing at speed $v . l_{c}$ depends on the energy of the solid-liquid interface:

$$
l_{c}=\frac{\gamma}{(\Delta c)^{2}(\partial f / \partial c)}
$$

where $\gamma$ is the line tension of the crystal-fluid interface, $\Delta c$ is the jump in concentration $c$ between the two phases, and $f$ is the chemical potential. The capillary length is difficult to calculate or measure directly, but it is essentially the interfacial thickness (4), which is at least one particle diameter in our 
system. Choosing $l_{c}=d$ to find the lower bound on the Mullins-Sekerka wavelength, and estimating the interfacial growth speed from our experiments, we obtain $\lambda_{s} \gtrsim 100 \mu \mathrm{m}$ on planar substrates for both $0.6-\mu \mathrm{m}$ and $1.0-\mu \mathrm{m}$-diameter particles. This is the characteristic width of dendrites if a kinetic instability is responsible for anisotropic growth. Crystals that are smaller than $\lambda_{s}$ will not show a dendritic instability, consistent with our observations on flat crystals.

Because the diffusion length $l_{d}$ and capillary length $l_{c}$ do not depend strongly on the substrate curvature, the Mullins-Sekerka wavelength $\lambda_{s}$ should not change significantly when the crystal grows on a curved surfaces. In fact, $\lambda_{s}$ is likely to be larger for a curved surface because of two effects. First, the free energy change associated with crystallization is smaller on a curved surface, because of the positive elastic energy cost associated with crystal growth. Thus the capillary length $l_{c}$ should be larger on a curved surface. Second, for crystals of the same diameter, the fluid particles have a smaller interfacial length to explore since the perimeter is smaller on a curved surface compared to a flat surface. The diffusion of fluid particles to interfacial irregularities is faster, which helps to stabilize the interface.

As a result, we do not expect the dendritic instability to appear on curved surfaces if the crystal sizes are smaller than $100 \mu \mathrm{m}$, which we consider a lower bound on the dendrite width. Our characteristic width of anisotropic growth is much smaller, even for our largest droplets: the biggest domain width is about $20 \mu \mathrm{m}$ for $1.0-\mu \mathrm{m}$ PS particles, and $25 \mu \mathrm{m}$ for $0.6-\mu \mathrm{m}$ PS particles. Thus we rule out a dendritic growth instability as the mechanism for anisotropic growth in our curved crystals.

\subsection{Estimation of spring constant}

As we discuss in the main text, we are able to fit the model to the observed domain widths to extract an effective spring constant for the interparticle interactions. This section describes our method. Our theoretical model predicts a constant width $w$ for an anisotropic crystallite grown on a curved surface. The stable domain width can be determined from our model by minimizing the net free energy of forming a ribbon-like domain as a function of domain width $w$ :

$$
w \approx \frac{4 \sqrt{2}}{\sqrt{3}}\left(\frac{\Delta f}{Y}\right)^{1 / 4} R
$$

where we have used rough estimates of $\Delta f$ and $Y$ based on the well depth $U_{m}$ and spring constant $k$ of the interparticle pair potential (Eq. 24 and Eq. 25). While we expect the well depth to be reasonably close to $U_{m}=4 k_{B} T$ for $1.0 \mu \mathrm{m}$ PS particles and $U_{m}=2.5 k_{B} T$ for $0.6 \mu \mathrm{m}$ particles, the theoretical estimate of the spring constant is less reliable, since it depends on the details of the short-ranged repulsive interactions between particles. However, we can estimate $k$ from the dependence of the domain width on droplet radius observed in the experiments.

Using a linear regression in Fig. $\mathrm{S} 4 \mathrm{~B}$, we extract a slope of 0.10 for the $w^{*} / d$-vs.- $R / d$ relationship for domains of $d=0.6 \mu \mathrm{m}$ particles, and a slope of 0.17 for domains of $d=1.0 \mu \mathrm{m}$ particles. Using Eq. 31, we estimate the spring constant as $k \approx 1 \times 10^{4} k_{B} T / \mu \mathrm{m}^{2}$ for $0.6 \mu \mathrm{m}$ particles and $k \approx 1 \times 10^{5} k_{B} T / \mu \mathrm{m}^{2}$ for $1.0 \mu \mathrm{m}$ particles. The spring constant estimate for $1.0 \mu \mathrm{m}$ PS particles is consistent with the estimate of $6 \times 10^{4} k_{B} T / \mu \mathrm{m}^{2}$ from the theoretical pair potential, Eq. 8 .

The value of $10^{5} k_{B} T / \mu \mathrm{m}^{2}$ for the spring constant is also consistent with that obtained from measurements of the ensemble probability of certain types of nonrigid clusters of $1.0 \mu \mathrm{m}$ PS particles under a similar depletion attraction potential (35). As discussed in ref. (35), the probability of observing a nonrigid clusters relative to all other clusters, including rigid and other nonrigid structures, depends on the spring constant of the potential through the vibrational entropy of the soft mode. The experimentally measured probability of a 9-particle nonrigid cluster of $1.0 \mu \mathrm{m}$ particles 


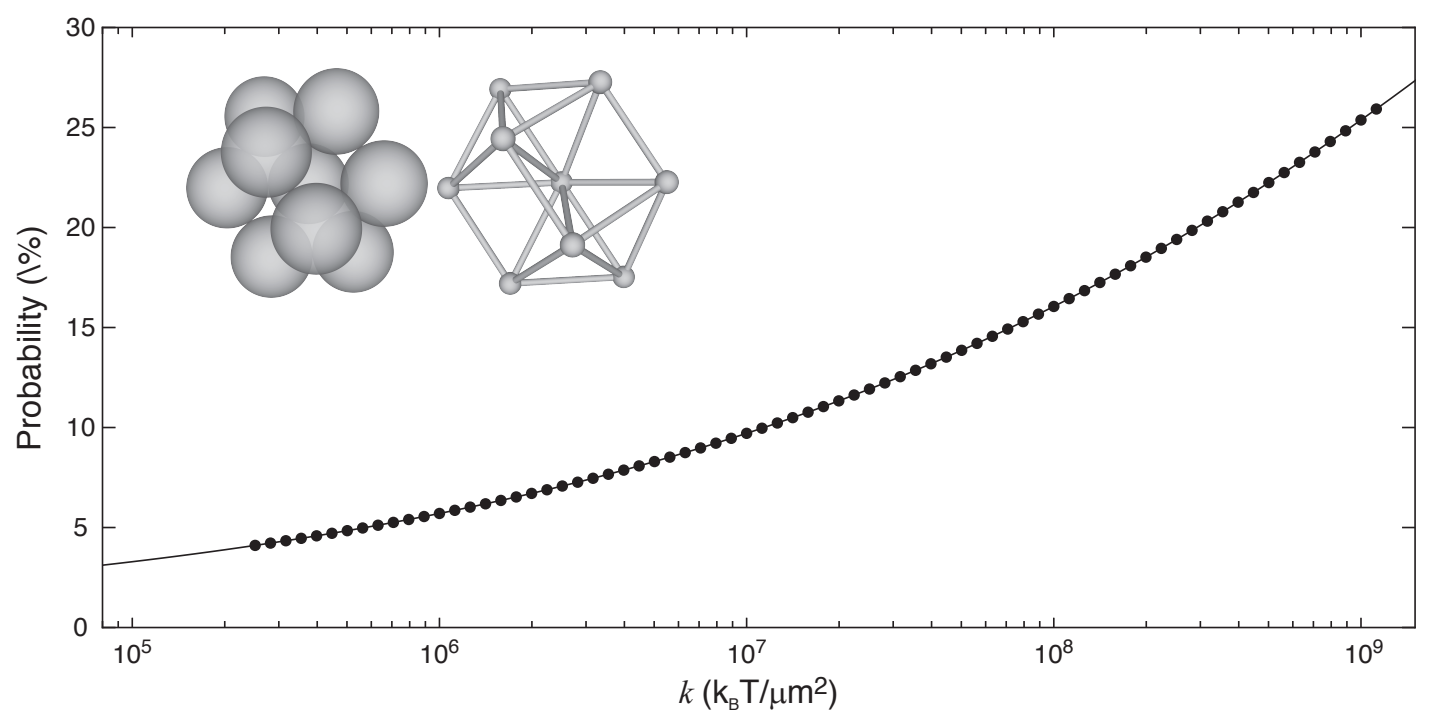

Fig. SM9. Theoretical equilibrium probability of observing a non-rigid cluster of 9 particles as a function of spring constant. The computer rendering shows the structure of the non-rigid cluster. The equilibrium probability (the black solid line) depends on the spring constant $k$ and is independent of the potential well depth. Black dots shows the region of $95 \%$ confidence interval of the experimental measurement of the equilibrium probability of observing the non-rigid cluster.

was $11 \%$, lying between $4 \%$ and $27 \%$ with a $95 \%$ confidence interval. The actual pair potential between PS particles can be approximated by a harmonic potential

$$
U(\Delta r)=\frac{1}{2} k \times \Delta r^{2}-U_{m},
$$

where $U_{m}$ is the strength of the potential, $\Delta r$ is the distance between particles around the equilibrium position, and $k$ is the spring constant. Using a statistical mechanical model to predict the probabilities of all other clusters (35) and a numerical calculation of the vibrational entropy associated with the soft mode (see Supplemental Information in ref. (35)), we can estimate the theoretical probability of observing the non-rigid cluster $P_{\text {theory }}$. Because the number of bonds is the same for all clusters, $P_{\text {theory }}$ depends only on the spring constant $k$ and is independent from the well depth $U_{m}$. Fig. SM9 shows how $P_{\text {theory }}$ varies with the spring constant $k$. The range $P_{\text {theory }}=4 \%-27 \%$ corresponds to spring constants between $2 \times 10^{5} k_{B} T / \mu \mathrm{m}^{2}$ and $1 \times 10^{9} k_{B} T / \mu \mathrm{m}^{2}$ for $1.0 \mu \mathrm{m}$ PS particles. 


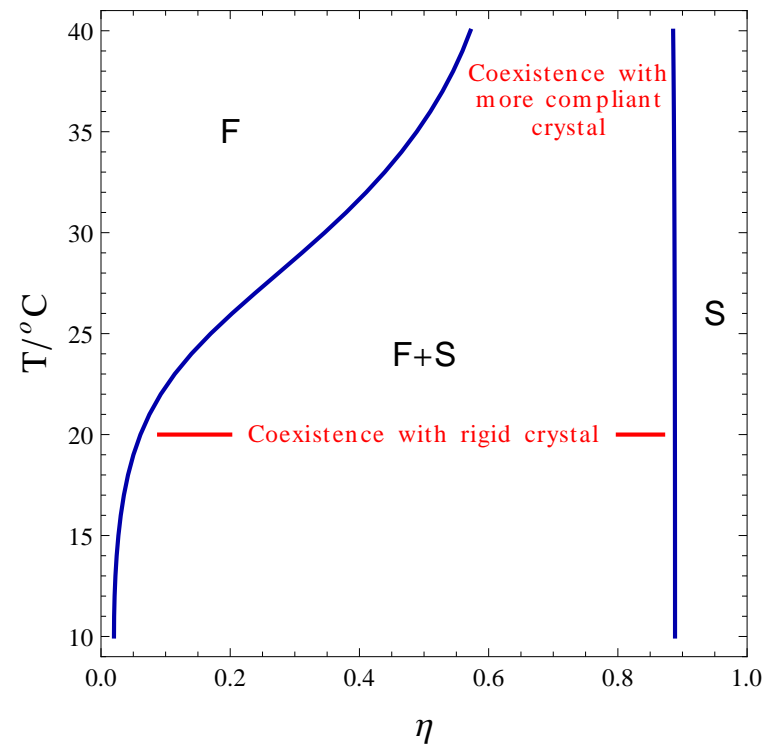

Fig. S1. Approximate phase diagram of the $2 \mathrm{D}$ colloidal system in flat space, using the hard-disk fluid and Einstein solid approximations. The temperature sets the depletant radius and volume fraction, as described in the text. Coexistence with a rigid crystal occurs below the shoulder in the coexistence curve, in a sublimation-like region of two-phase coexistence. 


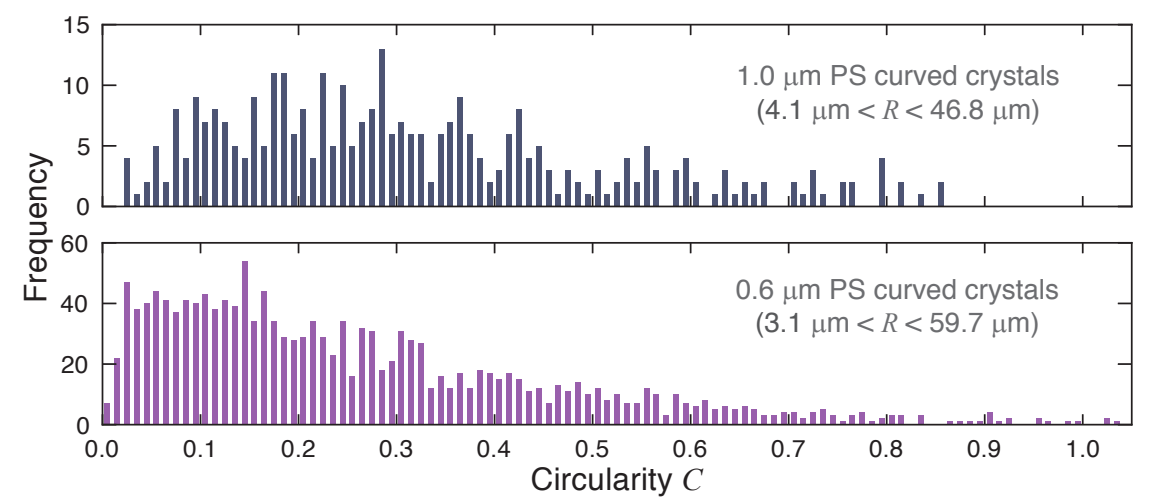

Fig. S2. Distribution of circularity $C$ of $1.0-\mu \mathrm{m}-\mathrm{PS}$ curved crystal domains (upper panel, 335 domains) and 0.6- $\mu \mathrm{m}$-PS curved crystal domains (lower panel, 1522 domains). 

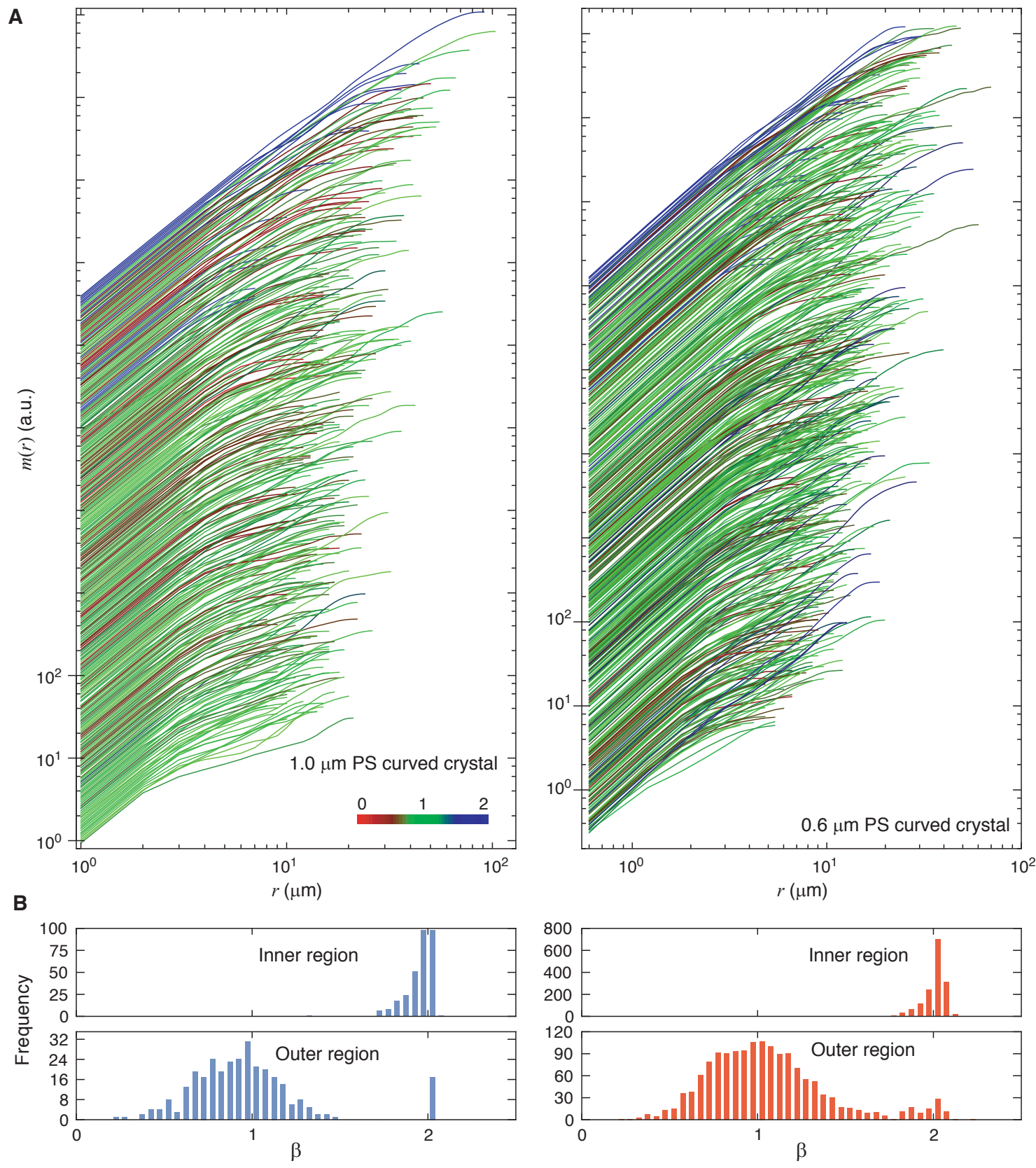

Fig. S3. Statistical results of mass dimension analysis. (A) Plot of measured $m(r)$ against $r$ for 306 1.0- $\mu \mathrm{m}$-PS curved crystal domains (left panel) and 507 0.6- $\mu \mathrm{m}$-PS curved crystal domains (right panel). The color of each line is set by the outer mass dimension obtained from the parametric fit (fit lines not shown). Lines are offset in the $y$-direction for clarity and ordered vertically by increasing size of the inner region $r_{\mathrm{I}}$ (obtained from the fit). Nearly all domains show a clear change in the mass scaling between small and large $r$. The predominance of green lines shows that most domains are indeed extended, displaying a mass dimension consistent with extended or branched shapes with near constant width. (B) Distribution of inner and outer mass dimensions for 306 1.0$\mu \mathrm{m}$-PS curved crystal domains (blue bars, left panel) and 1522 0.6- $\mu \mathrm{m}$-PS curved crystal domains (red bars, right panel). $\beta \approx 2.0$ for the inner regions and $\beta \approx 1.0$ for the outer regions. The mass dimension is therefore an independent measure showing that the crystals grown on curved surfaces are highly non-compact. 

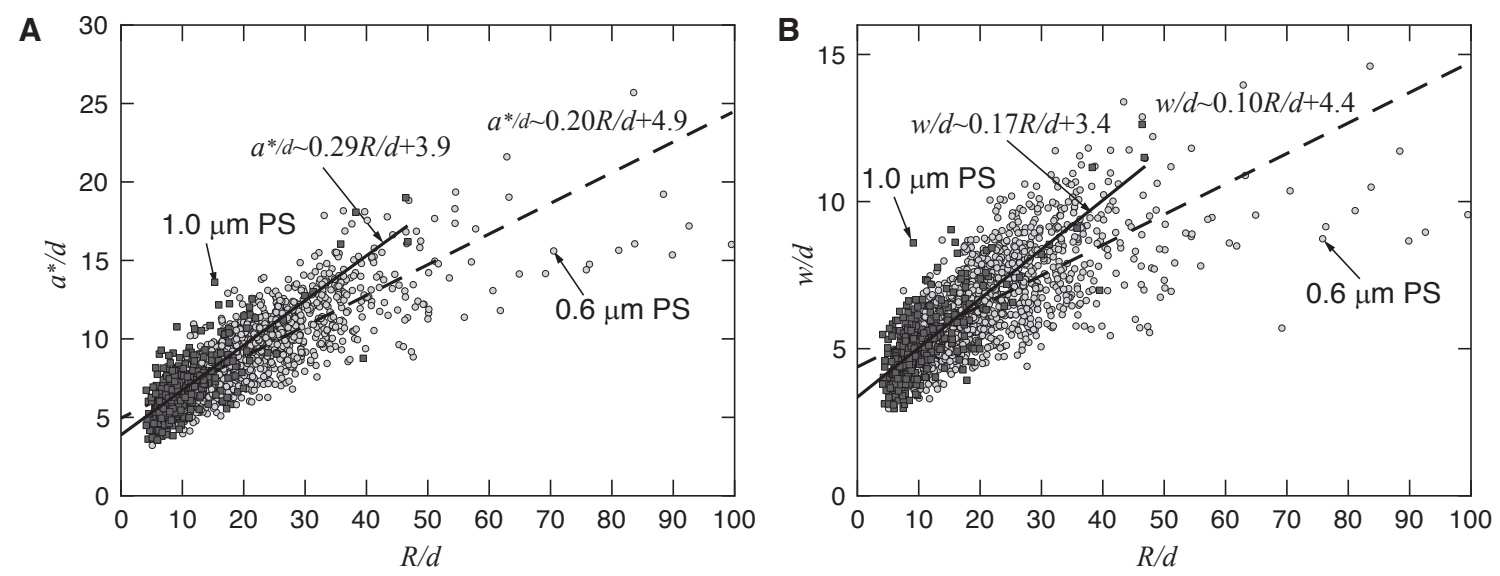

Fig. S4. Statistical results of morphological measurements of $1.0 \mu \mathrm{m}$ PS particles (dark squares, 335 domains) and $0.6 \mu \mathrm{m}$ PS particles (light circles, 1522 domains). (A) Curvature dependence of the largest inscribed circle diameter $a^{*} \sim R$. (B) Curvature dependence of the weighted average domain width along the backbones $w \sim R$. Both the inscribed disc diameter $a^{*}$ and the domain width $w$ increase with the radius of curvature $R$. This agrees with the prediction of the model in the main text. The scatter in the data reflects the variation in the initial particle densities, which leads to a variation in $\Delta f$ among experiments as well as stochastic effects that also affect the growth. 


\section{Movies}

Movie S1. Time-lapse images (confocal projections) of crystallization of $1 \mu \mathrm{m}$ particles on a droplet with $R=18.6 \mu \mathrm{m}$, showing growth of a single crystalline domain. Images correspond to Fig. 4 in the main text. Time stamps at upper left show time elapsed since sample preparation.

Movie S2. Time-lapse images (confocal projections) of crystallization of $1 \mu \mathrm{m}$ particles on a droplet with $R=12.0 \mu \mathrm{m}$, showing the growth and merging of multiple domains. This sample was prepared through a melt-quench method. 24 hours after sample preparation, the sample was heated until all the domains melted. Then the temperature was lowered $1{ }^{\circ} \mathrm{C}$ below the melting point, and the growth process was recorded. Time stamps at upper left show time elapsed since the quench.

Movie S3. As in Movie S2, but for a droplet with $R=23.7 \mu \mathrm{m}$.

Movie S4. As in Movie S2, but for a droplet with $R=38.2 \mu \mathrm{m}$. 


\section{References}

1. N. Steno, The prodromus of Nicolaus Steno's dissertation concerning a solid body enclosed by process of nature within a solid; an English version with an introduction and explanatory notes by John Garrett Winter (MacMillan, London, 1916).

2. I. Sunagawa, Crystals: Growth, Morphology, \& Perfection (Cambridge University Press, 2005).

3. A. T. Skjeltorp, Visualization and characterization of colloidal growth from ramified to faceted structures, Physical Review Letters 58, 1444 (1987).

4. J. S. Langer, Instabilities and pattern formation in crystal growth, Reviews of Modern Physics 52, 1 (1980).

5. K. G. Libbrecht, The physics of snow crystals, Reports on Progress in Physics 68, 855 (2005).

6. Materials and methods are available as supporting material on Science Online.

7. S. Asakura, F. Oosawa, On interaction between two bodies immersed in a solution of macromolecules, The Journal of Chemical Physics 22, 1255 (1954).

8. R. Roth, B. Götzelmann, S. Dietrich, Depletion forces near curved surfaces, Physical Review Letters 83, 448 (1999).

9. D. Nelson, F. Spaepen, Polytetrahedral order in condensed matter, Solid State Physics - Advances in Research and Applications 42, 1 (1989).

10. A. Haji-Akbari, M. Engel, A. S. Keys, X. Zheng, R. G. Petschek, P. Palffy-Muhoray, S. C. Glotzer, Disordered, quasicrystalline and crystalline phases of densely packed tetrahedra, $\mathrm{Na}$ ture 462, 773 (2009).

11. A. R. Bausch, M. J. Bowick, A. Cacciuto, A. D. Dinsmore, M. F. Hsu, D. R. Nelson, M. G. Nikolaides, A. Travesset, D. A. Weitz, Grain boundary scars and spherical crystallography, Science 299, 1716 (2003).

12. P. Lipowsky, M. J. Bowick, J. H. Meinke, D. R. Nelson, A. R. Bausch, Direct visualization of dislocation dynamics in grain-boundary scars, Nature Materials 4, 407 (2005).

13. W. T. M. Irvine, V. Vitelli, P. M. Chaikin, Pleats in crystals on curved surfaces, Nature 468, 947 (2010).

14. W. H. Roos, R. Bruinsma, G. J. L. Wuite, Physical virology, Nature Physics 6, 733 (2010).

15. I. R. Bruss, G. M. Grason, Non-euclidean geometry of twisted filament bundle packing, Proceedings of the National Academy of Sciences 109, 10781 (2012).

16. G. A. DeVries, M. Brunnbauer, Y. Hu, A. M. Jackson, B. Long, B. T. Neltner, O. Uzun, B. H. Wunsch, F. Stellacci, Divalent metal nanoparticles, Science 315, 358 (2007).

17. H. Bao, W. Peukert, R. K. Taylor, One-pot colloidal synthesis of plasmonic patchy particles, Advanced Materials 23, 2644 (2011).

18. R. Lipowsky, The conformation of membranes, Nature 349, 475 (1991).

19. J. Korlach, P. Schwille, W. W. Webb, G. W. Feigenson, Characterization of lipid bilayer phases by confocal microscopy and fluorescence correlation spectroscopy, Proceedings of the National Academy of Sciences 96, 8461 (1999).

20. A. Bandekar, S. Sofou, Floret-shaped solid domains on giant fluid lipid vesicles induced by $\mathrm{pH}$, Langmuir 28, 4113 (2012).

21. W. W. Mullins, R. F. Sekerka, Morphological stability of a particle growing by diffusion or heat flow, Journal of Applied Physics 34, 323 (1963).

22. W. W. Mullins, R. F. Sekerka, Stability of a planar interface during solidification of a dilute binary alloy, Journal of Applied Physics 35, 444 (1964).

23. S. Schneider, G. Gompper, Shapes of crystalline domains on spherical fluid vesicles, Euro- 
physics Letters 70, 136 (2005).

24. A. Y. Morozov, R. F. Bruinsma, Assembly of viral capsids, buckling, and the Asaro-GrinfeldTiller instability, Physical Review E 81, 041925 (2010).

25. Y. Chushak, A. Travesset, Solid domains in lipid vesicles and scars, Europhysics Letters $\mathbf{7 2}$, 767 (2005).

26. D. R. Nelson, Defects and Geometry in Condensed Matter Physics (Cambridge University Press, 2002).

27. C. Majidi, R. S. Fearing, Adhesion of an elastic plate to a sphere, Proceedings of the Royal Society A: Mathematical, Physical and Engineering Science 464, 1309 (2008).

28. R. F. Garmann, M. Comas-Garcia, A. Gopal, C. M. Knobler, W. M. Gelbart, The assembly pathway of an icosahedral single-stranded RNA virus depends on the strength of inter-subunit attractions, Journal of Molecular Biology advance online publication (2013).

29. W. Klug, W. Roos, G. Wuite, Unlocking internal prestress from protein nanoshells, Physical Review Letters 109, 168104 (2012).

30. A. Luque, D. Reguera, A. Morozov, J. Rudnick, R. Bruinsma, Physics of shell assembly: Line tension, hole implosion, and closure catastrophe, The Journal of Chemical Physics 136, 184507 (2012).

31. J. D. Bernal, The Bakerian lecture, 1962. The structure of liquids, Proceedings of the Royal Society of London. Series A. Mathematical and Physical Sciences 280, 299 (1964).

32. P. Robert, Temperature-sensitive aqueous microgels, Advances in Colloid and Interface Science 85, 1 (2000).

33. J. W. Goodwin, J. Hearn, C. C. Ho, R. H. Ottewill, Studies on the preparation and characterisation of monodisperse polystyrene laticee, Colloid \& Polymer Science 252, 464 (1974).

34. J. C. Crocker, D. G. Grier, Methods of digital video microscopy for colloidal studies, Journal of Colloid and Interface Science 179, 298 (1996).

35. G. Meng, N. Arkus, M. P. Brenner, V. N. Manoharan, The Free-Energy landscape of clusters of attractive hard spheres, Science 327, 560 (2010).

36. A. D. Dinsmore, E. R. Weeks, V. Prasad, A. C. Levitt, D. A. Weitz, Three-Dimensional confocal microscopy of colloids, Applied Optics 40, 4152 (2001).

37. E. Jones, T. Oliphant, P. Peterson, et al., SciPy: Open source scientific tools for Python, http://www.scipy.org (2001-).

38. E. R. Dougherty, R. A. Lotufo, Hands-on morphological image processing (Spie Press, 2003).

39. H. Xia, P. G. Tucker, Proceedings of the 18th International Meshing Roundtable (SpringerVerlag, 2009), pp. 247-265.

40. A. Vrij, Polymers at interfaces and the interactions in colloidal dispersions, Pure and Applied Chemistry 48, 471 (1976).

41. W. B. Russel, D. A. Saville, W. R. Schowalter, Colloidal Dispersions (Cambridge University Press, 1992).

42. M. Dijkstra, Phase behavior of hard spheres with a short-range yukawa attraction, Physical Review E 66, 021402 (2002).

43. P. Bolhuis, M. Hagen, D. Frenkel, Isostructural solid-solid transition in crystalline systems with short-ranged interaction, Physical Review E 50, 4880 (1994).

44. J. Serrano-Illán, G. Navascués, E. Velasco, L. Mederos, Self-consistent nonperturbative theory: Treatment of colloidal-type interactions, The Journal of Chemical Physics 119, 1510 (2003).

45. T. Chou, D. R. Nelson, Dislocation-mediated melting near isostructural critical points, Physical Review E 53, 2560 (1996). 
46. A. Santos, M. López de Haro, S. Bravo Yuste, An accurate and simple equation of state for hard disks, The Journal of Chemical Physics 103, 4622 (1995).

47. H. Reiss, H. L. Frisch, J. L. Lebowitz, Statistical mechanics of rigid spheres, The Journal of Chemical Physics 31, 369 (1959).

48. E. Helfand, H. L. Frisch, J. L. Lebowitz, Theory of the two- and one-dimensional rigid sphere fluids, The Journal of Chemical Physics 34, 1037 (1961).

49. J. A. Zollweg, G. V. Chester, P. W. Leung, Size-dependent properties of two-dimensional solids, Physical Review B 39, 9518 (1989).

50. M. Engel, J. A. Anderson, S. C. Glotzer, M. Isobe, E. P. Bernard, W. Krauth, Hard-disk equation of state: First-order liquid-hexatic transition in two dimensions with three simulation methods, Physical Review E 87, 042134 (2013).

51. H. S. Seung, D. R. Nelson, Defects in flexible membranes with crystalline order, Physical Review A 38, 1005 (1988).

52. P. R. t. Wolde, D. Frenkel, Enhancement of Protein Crystal Nucleation by Critical Density Fluctuations, Science 277, 1975 (1997).

53. J. Lutsko, G. Nicolis, Theoretical Evidence for a Dense Fluid Precursor to Crystallization, Physical Review Letters 96, 046102 (2006).

54. J.-P. Hansen, I. R. McDonald, Theory of simple liquids (Access Online via Elsevier, 1990).

55. D. G. Chae, F. H. Ree, T. Ree, Radial Distribution Functions and Equation of State of the Hard-Disk Fluid, The Journal of Chemical Physics 50, 1581 (1969).

56. G. Tarjus, F. Sausset, P. Viot, Statistical mechanics of liquids and fluids in curved space, arXiv preprint arXiv: 1005.2684 (2010).

57. S. Lishchuk, Equation of state of the hard-disk fluid on a sphere from Percus-Yevick equation, Physica A: Statistical Mechanics and its Applications 369, 266 (2006).

58. L. D. Landau, L. P. Pitaevskii, E. Lifshitz, A. M. Kosevich, Theory of Elasticity, Third Edition: Volume 7 (Butterworth-Heinemann, 1986). 